\title{
Development and validation of ferroptosis-related IncRNAs signature for hepatocellular carcinoma
}

\author{
Jiaying Liang Equal first author, 1, 2 , Yaofeng Zhi ${ }^{\text {Equal first author, 1, } 2 \text {, Wenhui Deng }}{ }^{3}$, Weige Zhou ${ }^{1}$, Xuejun Li ${ }^{1}$, Zheyou Cai ${ }^{1}$, \\ Zhijian Zhu ${ }^{1}$, Jinxiang Zeng ${ }^{1}$, Wanlan Wu ${ }^{1}$, Ying Dong ${ }^{1}$, Jin Huang ${ }^{4}$, Yuzhuo Zhang ${ }^{1,2}$, Shichao Xu ${ }^{1,2}$, Yixin Feng ${ }^{1,}$ \\ ${ }^{2}$, Fuping Ding ${ }^{\text {Corresp., } 5}$, Jin Zhang ${ }^{\text {Corresp. 1, } 2}$ \\ ${ }^{1}$ Guangzhou University of Chinese Medicine, School of Basic Medical Sciences, Guangzhou, China \\ 2 Guangzhou University of Chinese Medicine, Research Center of Integrative Medicine,School of Basic Medical Sciences, Guangzhou, China \\ 3 Guangzhou University of Chinese Medicine, The fourth Affiliated Hospital of Guangzhou University of Chinese Medicine, Shenzhen, China \\ 4 Guangzhou University of Chinese Medicine, Clinic of Guangzhou University of Chinese Medicine, Guangzhou, China \\ 5 Guangzhou University of Chinese Medicine, School of Nursing, Guangzhou, China \\ Corresponding Authors: Fuping Ding, Jin Zhang \\ Email address: hldfp@gzucm.edu.cn, zhjin@gzucm.edu.cn
}

Background. Hepatocellular carcinoma (HCC) with high heterogeneity is one of the most frequent malignant tumors throughout the world. However, there is no research to establish ferroptosis-related IncRNAs (FRIncRNAs) signature for the patients with HCC. Therefore, this study was designed to establish a novel FRIncRNAs signatures to preferable predict the survival of patients with HCC.

Method. The expression profiles of IncRNAs were acquired from The Cancer Genome Atlas (TCGA) and Gene Expression Omnibus (GEO) database. FRIncRNAs co-expressed with ferroptosis-related gene were utilized to establish signature. Cox regression was used to construct a novel three FRIncRNAs signature in TCGA cohort, which was verified in GEO validation cohort.

Results. Three differently expressed FRIncRNAs significantly associated with prognosis of HCC were identified, which composed a novel FRIncRNAs signature. According to the FRIncRNAs signature, the patients with HCC could be divided into low- and high-risk groups. Patients with HCC in high-risk group displayed shorter overall survival (OS) contrasted with those in low-risk group $(P<0.001$ in TCGA cohort and $P=0.045$ in GEO cohort). This signature could serve as a significantly independent predictor in Cox regression (multivariate $H R>1, P<0.001$ ), which was verified to a certain extent in GEO cohort (univariate $H R>1, P<0.05$ ). Meanwhile, it was also a useful tool in predicting survival among each stratum of gender, age, grade, stage, and etiology,etc. This signature was connected with immune cell infiltration (i.e., Macrophage, Myeloid dendritic cell, and Neutrophil cell, etc.) and immune checkpoint blockade targets (PD-1, CTLA-4, and TIM-3).

Conclusion. The three FRIncRNAs might be potential therapeutic targets for patients, and their signature could be utilized for prognostic prediction in HCC. 
1 Development and validation of ferroptosis-related IncRNAs signature for

\section{2 hepatocellular carcinoma}

3

4 Jiaying Liang ${ }^{\# 1,2}$, Yaofeng Zhi ${ }^{\sharp 1,2}$, Wenhui Deng ${ }^{3}$, Weige Zhou ${ }^{1}$, Xuejun $\mathrm{Li}^{1}$, Zheyou Cai ${ }^{1}$, Zhijian $5 \mathrm{Zhu}^{1}$, Jinxiang Zeng ${ }^{1}$, Wanlan $\mathrm{Wu}^{1}$, Ying Dong ${ }^{1}$, Jin Huang ${ }^{4}$, Yuzhuo Zhang ${ }^{1,2}$, Shichao $\mathrm{Xu}^{1,2}$, 6 Yixin Feng ${ }^{1,2}$, Fuping Ding ${ }^{5}$, Jin Zhang ${ }^{1,2}$

$8{ }^{1}$ School of Basic Medical Sciences, Guangzhou University of Chinese Medicine, Guangzhou, 9 China.

2 Research Center of Integrative Medicine, School of Basic Medical Sciences, Guangzhou University of Chinese Medicine, Guangzhou, China.

3 The fourth Affiliated Hospital of Guangzhou University of Chinese Medicine, Guangzhou University of Chinese Medicine, Shenzhen, China. Medicine, Guangzhou, China.

\# Contributed equally.

\section{ABSTRACT}

Background. Hepatocellular carcinoma (HCC) with high heterogeneity is one of the most frequent malignant tumors throughout the world. However, there is no research to establish ferroptosis-related lncRNAs (FRlncRNAs) signature for the patients with HCC. Therefore, this study was designed to establish a novel FRlncRNAs signatures to preferable predict the survival of patients with HCC.

Method. The expression profiles of lncRNAs were acquired from The Cancer Genome Atlas (TCGA) and Gene Expression Omnibus (GEO) database. FRlncRNAs co-expressed with ferroptosis-related gene were utilized to establish signature. Cox regression was used to construct a novel three FRlncRNAs signature in TCGA cohort, which was verified in GEO validation cohort. 
Results. Three differently expressed FRlncRNAs significantly associated with prognosis of HCC were identified, which composed a novel FRlncRNAs signature. According to the FRlncRNAs signature, the patients with HCC could be divided into low- and high-risk groups. Patients with HCC in high-risk group displayed shorter overall survival (OS) contrasted with those in low-risk group ( $P<0.001$ in TCGA cohort and $P=0.045$ in GEO cohort). This signature could serve as a significantly independent predictor in Cox regression (multivariate HR $>1, P<0.001$ ), which was verified to a certain extent in GEO cohort (univariate HR $>1, P<0.05$ ). Meanwhile, it was also a useful tool in predicting survival among each stratum of gender, age, grade, stage, and etiology, etc. This signature was connected with immune cell infiltration (i.e., Macrophage, Myeloid dendritic cell, and Neutrophil cell, etc.) and immune checkpoint blockade targets (PD-1, CTLA-4, and TIM-3).

Conclusion. The three FRIncRNAs might be potential therapeutic targets for patients, and their signature could be utilized for prognostic prediction in HCC.

Keywords: hepatocellular carcinoma, ferroptosis, immunotherapy, IncRNA, gene signature

\section{INTRODUCTION}

Hepatocellular carcinoma (HCC), which is the second frequent cause of death in human cancers throughout the world, is one of the most familiar malignant tumors (Llovet et al. 2016). It is evaluated that $\sim 841,000$ new cases are diagnosed with $\mathrm{HCC}$ and approximately 781,631 patients would die of HCC in 2018 (Bray et al. 2018). For early-stage patients, radiofrequency local ablation, partial hepatectomy and liver transplantation are the major therapies and about $70 \%$ of patients will relapse within five years after operation (European Association for the Study of the Liver. Electronic address \& European Association for the Study of the 2018). Immune checkpoint inhibitors have been proved to be effective strategies for the therapy of advanced HCC, but their effectiveness still needed to be further improved (Yang et al. 2019). Despite the new advance in early detection, and drug development, the clinical outcomes of advanced cases remain unsatisfactory. The 5-year survival rate of local $\mathrm{HCC}$ is $30.5 \%$, and that of distant metastasis was less than 5\% (Oweira et al. 2017). To improve clinical outcomes and reduce the burden of cases, it is urgent to identify novel effective molecular markers and ameliorate prediction of HCC prognosis.

Ferroptosis is an iron-dependent modality of regulated cell death driven by the malignant accumulation of lipid peroxidation (Dixon et al. 2012; Stockwell et al. 2017). Recently, the induction of ferroptosis has been listed as a promising therapeutic strategy, especially suitable for malignant tumor that respond to resistance in traditional treatments (Hassannia et al. 2019; Liang et al. 2019). A large number of experiment studies had indicated that ferroptosis-related genes 
68

69

played a vital role in HCC (Jennis et al. 2016; Louandre et al. 2015; Sun et al. 2016a; Sun et al. 2016b; Yuan et al. 2016).

Long non-coding RNA (lncRNA) with a minimum length of about 200 nucleotides are autonomous transcriptional RNA which does not encode proteins (Cech \& Steitz 2014). LncRNAs have been proved to be abnormally expressed in multiple cancers, and aberrant lncRNAs have been reported to serve as prognostic indicators in various cancers including HCC (Ai et al. 2020; He et al. 2019; Li et al. 2019; Wang et al. 2018; Ye et al. 2019; Zeng et al. 2020; Zhao et al. 2017). One recent study revealed that ferroptosis-related lncRNAs signature was associated with the prognosis of patients with head and neck squamous cell carcinoma (Tang et al. 2021). However, there is little research on ferroptosis-related lncRNAs correlated with HCC patient prognosis. Therefore, this study aims to establish a novel ferroptosis-related lncRNAs (FRlncRNAs) signature in predicting the prognosis of patients with HCC, hoping to improve current diagnosis, treatment, follow-up and prevention strategies of HCC.

\section{MATERIALS AND METHODS}

\section{Data source and clinical information}

The RNA sequencing (RNA-seq) data together with relevant clinical data were accessed from The Cancer Genome Atlas (TCGA) database (https://portal.gdc.cancer.gov/). An overview of the clinical information and source file of the patients with HCC can be found in Table 1 and Table S1. Notably, those patients with follow-up time greater than one month were used for the study. Totally, 374 Hepatocellular carcinoma (HCC) patients were available for further analysis. The GSE14520 dataset was acquired from GEO database (http://www.ncbi.nlm.nih.gov/geo/), containing 488 patients with HCC. A total of 259 ferroptosis-related genes (Marker: 111; Driver: 108; Suppressor: 69) were identified from FerrDb Database (Zhou \& Bao 2020) (FerrDb, http://www.zhounan.org/ferrdb/; Table S2). The TCGA dataset was utilized for training cohort while the GSE14520 dataset was validation cohort.

\section{LncRNAs and ferroptosis-related genes data processing}

The "limma" package was employed to select differentially expressed ferroptosis-related genes, which were visualized through the volcano and heatmaps. Then, we carried out functional enrichment analysis (Gene Ontology (GO) and Kyoto Encyclopedia of Genes and Genomes (KEGG)) to determine the major biological attributes. The "GOplot" package was utilized to visualize enrichment terms.

To calculate the correlation between candidate ferroptosis-related lncRNAs (FRlncRNAs) and differentially expressed ferroptosis-related genes utilizing Pearson correlation. The coefficient $P$ 
$102<0.001$ and $\left|\mathrm{R}^{2}\right|>0.3$ were regarded to be FRlncRNAs. Finally, Cytoscape software was utilized

103 to draw co-expression network of prognostic FRlncRNAs and ferroptosis-related genes.

\section{Construction of prognostic FRIncRNAs signature}

105 First, the FRlncRNAs associated with prognosis were assessed using univariate Cox regression in training cohort. Then, FRlncRNAs with $P \leq 0.05$ were included into multivariate Cox regression for the construction of FRlncRNAs signature. The formula utilized was as follows: risk score of

FRlncRNAs signature $\left.=\sum_{i} \operatorname{Coefficient}_{(F R \operatorname{lncRNAs}}\right) * \operatorname{Expression}\left(F R \operatorname{lncRNAs_{i}}\right)$. To stratify

109

110

111

112

patients into low- or high-risk groups, the best cut-off of the FRlncRNAs signature was identified applying receiver operating characteristic (ROC) curve at 1 year for overall survival (OS). Survival analysis between the two risk groups were assessed by Kaplan-Meier (KM) and compared using log-rank statistical methods.

Nomogram was utilized to predict 1, 3 years, and 5 years survival for the patients with HCC. ROC curves and Calibration curves were utilized to explore the accuracy of model based on training cohort. Then, we adjusted other clinical features in independent prognostic analysis in order to confirm whether the FRlncRNAs signature was an independent indicator to predict the prognosis of patient with HCC.

\section{Validation of the FRIncRNAs signature}

The GEO cohort was enrolled to verify the robustness of model established from training cohort. The FRlncRNAs signature was calculated based on validation cohort. Then, survival analysis and Cox regression were utilized to evaluate whether the FRIncRNAs signature was significantly connected with OS in validation cohort. The ROC curves were established to assess whether the novel model could accurately predict patient survival.

\section{Gene set enrichment analysis}

Gene set enrichment analysis (GSEA, http://www.broadinstitute.org/gsea/index.jsp) was utilized to investigate functional phenotypes differences between the two risk groups (high- and low-risk groups). In this research, we carried out functional enrichment of FRlncRNAs signature, and visualized the pathway closely related to immune and tumorigenesis development. The reference gene sets contained "c7.all.v7.2.symbols.gmt [Immunologic signatures] and h.all.v7.2.symbols.gmt [cancer hallmarks]".

\section{Immune correlation analysis}

The CIBERSORT (Charoentong et al. 2017; Newman et al. 2015), EPIC (Racle et al. 2017), ESTIMATE (Yoshihara et al. 2013), MCP counter (Shi et al. 2020), QUANTISEQ (Finotello et al. 2019), TIMER (Li et al. 2017), and single-sample gene set enrichment analysis (ssGSEA) (Yi et al. 2020) algorithms were used to infer the relative content of tumor-infiltrating immune cells (TIICs) between two risk group based on FRlncRNAs signature. The heatmap was utilized to 
137

138

139

visualize the differences of TIICs abundance under different algorithms. Besides, the correlation analysis between the abundance of TIICs and FRlncRNAs signature was utilized to reveal the potential role of FRlncRNAs signature on the immunologic features based on TIMER results.

The expression of immune checkpoint gene might be related to treatment responses of immune checkpoint inhibitors (ICIs) (Goodman et al. 2017). Thus, we investigated six ICIs: programmed death 1 (PD-1) and its ligand 1 (PD-L1), ligand 2 (PD-L2), indoleamine 2,3-dioxygenase 1 (IDO1), cytotoxic T-lymphocyte antigen 4 (CTLA-4), and T cell immunoglobulin and mucin domaincontaining protein-3 (TIM-3) in HCC (Kim et al. 2017; Nishino et al. 2017; Zhai et al. 2018). We analyzed the spearman correlation between the ICIs and the signature, which aimed to investigate the potential role of FRlncRNAs signature in immune checkpoint blockade therapy.

\section{Statistical analysis}

All the statistical analyses were conducted using R language (version 4.0). KM analysis with logrank test from "survival" package was applied to compare the survival difference among two risk groups. In order to evaluate the prognostic value of the novel FRlncRNAs signature, univariate and multivariate analyses were conducted through Cox proportional hazards regression model. Stratification analysis was implemented based on age $(\geq 65$ and $<65$ years), gender (male and female), stage (stage 1-2 and stage 3-4), grade (grade 1-2 and grade 3-4), etiology (Alcoholic liver disease and nonalcoholic fatty liver disease; $\mathrm{HBV} / \mathrm{HCV}$ and non-HBV/HCV), Radiotherapy (receive radiotherapy and no receive radiotherapy), and family history (have family history of cancer and no family history). GSEA was applied to differentiate between two risk groups of functional annotations. Statistical tests were bilateral, with $P$ value $\leq 0.05$ indicated statistically significant differences.

\section{RESULTS}

\section{Differentially expressed ferroptosis-related genes}

After extracting the expression values of 259 ferroptosis-related genes in the patient with HCC, 69 up-regulated genes and 13 down-regulated genes were authenticated (FDR $<0.05, \log 2 \mathrm{FC}>1$; Table S3). The differentially expressed ferroptosis-related genes were visualized by volcano and heatmaps (Figs. 1A-B). The GO enrichment revealed that these differentially expressed ferroptosis-related genes mainly participated in cellular response to chemical stress, response to oxidative stress, and carboxylic acid transport among others (Fig. 1C). The major KEGG pathways included Ferroptosis, VEGF signaling pathway, Arachidonic acid metabolism and some cancerrelated signaling pathways (Fig. 1D; Table S4).

\section{Identification of prognostic FRIncRNAs and an FRIncRNAs signature}

A total of 1,184 FRIncRNAs were identified in the training cohort through the correlation analysis 
172 between differentially expressed ferroptosis-related genes and $\operatorname{lncRNAs}\left(\left|\mathrm{R}^{2}\right|>0.3\right.$ and $P<0.001$;

173 Table S5). Among them, 32 FRlncRNAs co-existed in training cohort and validation cohort were 174 used for subsequent model construction and validation. The batch effects from different cohorts

175 were corrected by combat function in "sva" package. Cox regression were applied to screen prognostic FRlncRNAs. In accordance with univariate cox results, eight FRlncRNAs had potential prognostic value for the patients with HCC ( $\mathrm{P}<0.05$, Fig. 2A, Table S6). As shown in Table S6, eight FRlncRNAs (RHPN1-AS1, SNHG17, DGCR11, MAPKAPK5-AS1, PVT1, PART1, LINC00339 and HCG18) were discovered to be harmful prognostic indicators.

Subsequently, multivariate Cox regression found that three FRlncRNAs were associated with prognostic for the patients with HCC (Table S6). The three FRlncRNAs were utilized to establish a FRlncRNAs signature. The risk score was estimated using the following formula: risk score of FRlncRNAs signature $=(0.89773 *$ RHPN1-AS1 $)+\left(0.48558^{*}\right.$ MAPKAPK5-AS1 $)+$ $(0.55674 *$ PART1). The optimal cut-off point of risk score was considered to be 1.038 through ROC curve. Based on this cut-off point, the patients with HCC were classified into high- or lowrisk group (Fig. 2B). The relationship between the three prognostic FRlncRNAs and co-expressed mRNA was shown in Figure 2C and Figure 2D. The risk score was significantly relevant to OS of patients, where OS in high-risk group possessed shorter than those in low-risk group $(P<0.001$, Fig. 3A). Concurrently, the AUC of the FRlncRNAs signature was 0.719, showing great performance in contrast to other traditional clinical pathological features in predicting the prognosis of patients with HCC (Fig. 3B). The survival status plot showed that risk score of patients was inversely proportional to their survival rate. Besides, the risk heatmap demonstrated that the expression of three FRlncRNAs had positive correlation with the risk levels (Fig. 3C). The areas under the ROC (AUC) values corresponding to 1, 3, and 5 years were $0.711,0.649$ and 0.632 , respectively (Fig. 3D).

\section{The FRIncRNAs signature was an independent prognostic indicator}

Univariate independent prognostic analysis revealed that risk score was a prognostic factor and significantly associated with worse survival ( $\mathrm{HR}=1.732,95 \% \mathrm{CI}: 1.324-2.267 ; P<0.001)$ (Table 2, Fig. 4A). Moreover, after adjusting other available clinical parameters such as age, gender, stage, grade, etiology, radiotherapy, and family history, our signature still maintained an independent prognostic factor in multivariate independent analysis $(\mathrm{HR}=1.721,95 \% \mathrm{CI}=$ 1.280-2.314; $P<0.001$ ) (Table 2, Fig. 4B). As indicated in the nomogram, three-FRlncRNAbased signature was the largest contribution to OS of each period in HCC (Fig. 4C). The Calibration displayed that the FRlncRNAs signature possessed high accuracy (Figs. 4D-4F).

\section{Stratification analyses}

In training cohort, stratification analysis was conducted based on the clinicopathological features of HCC (e.g., gender, age, grade, stage, etiology, radiotherapy, and family history). As a result, 
208

209

210

211

212

213

214

215

216

217

218

219

220

221

222

223

224

225

226

227

228

229

230

231

232

233

234

235

236

237

238

239

240

241

242

243

the FRlncRNAs signature was still closely associated with worse survival in male, older $(\geq 65$ years) or younger $(<65$ years), advanced grade (Grade 3-4) or early grade (Grade 1-2) and advanced stage (Stage 3-4) or early stage (Stage 1-2) patients (all $P<0.05$; Figs. 5A-F), which indicated that FRlncRNAs signature based on risk grouping could serve as a useful tool for predicting HCC survival among each stratum of gender, age, grade, and stage. Meanwhile, this signature can also be utilized as a potential prognostic tool for HCC patients with alcoholic or nonalcoholic liver disease, no family history of cancer, HBV-positive, HBV- and HCV-negative, and no radiotherapy (Figs. 5G-M).

\section{Validation of the FRIncRNAs signature}

In validation cohort, the risk score of FRIncRNAs signature was estimated refer to the previous formula. The cut-off point of the risk score of the validation cohort was consistent with that of the training cohort (Cutoff $=1.038)$. The signature was also statistically associated with OS of patients with HCC $(P=4.504 \mathrm{e}-02$, Fig. 6A). Univariate independent prognostic analysis revealed that FRIncRNAs signature acted as an independent prognostic factor (HR of risk score $=1.410,95 \%$ CI: 1.054-1.887, $P<0.05$, Table 2, Fig. 6B). Notably, after controlling gender, age, TNM stage, and CLIP stage, FRlncRNAs signature was no longer a prognostic factor in multivariate analysis $(\mathrm{HR}=1.049,95 \% \mathrm{CI}=0.764-1.439, P>0.05)$, which indicated that more independent cohorts needed to be included for validation (Table 2, Fig. 6C). The AUC values were more than 0.65 at 1 year, 2 years and 3 years, which showed that the FRlncRNAs signature established from training cohort had powerful accuracy and robustness (Fig. 6D).

The FRIncRNAs signature mediated DNA repair, glycolysis, MYC targets, P53 pathway and PI3K AKT mTOR signaling

GSEA was utilized to explore the potential biological mechanisms of the FRlncRNAs signature involved in HCC progression. The results of cancer hallmarks indicated that DNA repair, glycolysis, MYC targets, P53 pathway and PI3K AKT mTOR signaling were activated by the high-risk group of the FRlncRNAs signature. While adipogenesis, bile acid metabolism, coagulation, fatty acid metabolism and xenobiotic metabolism were activated by the low-risk group (Fig. 7A). Moreover, the signature also regulated many immunologic features within immune system, such as BCL6 high versus low TFH CD4 T cell down, cent memory CD4 T cell versus TH2 down etc., which indicated that the FRlncRNAs signature was implicated in immunity-related regulation (Fig. 7B).

\section{Correlation of FRIncRNAs signature with TICIs and immune checkpoint blockade (ICB)} molecule

Many immunologic features were found to be regulated by FRlncRNAs signature according to the above results. Therefore, the signature was further explored whether it was associated with TIICs based on TIMER results. The results indicated that this signature was most significantly positive 
244

245

246

247

248

249

250

251

252

253

254

255

256

257

258

259

260

261

262

263

264

265

266

267

268

269

270

271

272

273

274

275

276

277

278

correlation with immune infiltration of Neutrophil cells ( $\mathrm{COR}=0.312, P<0.001)$, Myeloid cells $(\mathrm{COR}=0.268, P<0.001), \mathrm{CD} 4+\mathrm{T}$ cells $(\mathrm{COR}=0.203, P<0.001), \mathrm{B}$ cells $(\mathrm{COR}=0.130, P=$ 0.016 ), and Macrophage cells (COR $=0.124, P=0.021$; Figs. $8 \mathrm{~A}-\mathrm{F})$. The heatmap of immune responses based on CIBERSORT, EPIC, ESTIMATE, MCP counter, QUANTISEQ, TIMER and ssGSEA algorithms was displayed in Figure 9. These findings powerfully indicated that this FRlncRNAs signature was related to immune cell infiltration in HCC.

Tumor immunotherapy utilizing ICB had gradually become a promising strategy for therapy of advanced HCC (Sangro et al. 2021; Wing-Sum Cheu \& Chak-Lui Wong 2021; Zongyi \& Xiaowu 2020). In training cohort, we carried out the association between the FRIncRNAs signature and six common ICB therapy-related targets (PD-1, PD-L1, PD-L2, TIM-3, IDO1, and CTLA-4) to explore the potential role of FRIncRNAs signature in the immunotherapy of ICB in the patients with HCC. The results showed that the FRIncRNAs signature was positively related to PD $-1(R=$ $0.17, P=0.0019)$, CTLA-4 $(R=0.19, P=<0.001)$, and TIM-3 $(R=0.16, P<0.001$; except for IDO1, PD-L1, and PD-L2), revealing that the FRlncRNAs signature might play vital roles in assessment of response to ICB immunotherapy in the patients with HCC (Figs. 8G-I and Fig. S1). Meanwhile, the expression levels of PD-1, CTLA-4 and TIM-3 were significantly higher in highrisk group contrasted with those in low- group (Figs. 8J-L).

\section{DISCUSSION}

Due to the unique molecular features such as genomic and genetic diversities, HCC was considered as a highly heterogeneous malignant tumor (Cancer Genome Atlas Research Network. Electronic address \& Cancer Genome Atlas Research 2017; Schulze et al. 2016). Studies found that IncRNAs play important roles in the prognosis of HCC, which could become potential and effective molecular targets in the treatment of HCC (DiStefano 2017; Wei et al. 2019). It could be seen from previous studies that lncRNAs participate in many biological processes such as immune response, autophagy, inflammation, and metabolism, etc (Carpenter \& Fitzgerald 2018; Frankel et al. 2017; Majidinia \& Yousefi 2016; Mathy \& Chen 2017). At present, emerging studies revealed that some lncRNAs could play significant roles in regulating occurrence and development of disease by promoting ferroptosis ( $\mathrm{Lu}$ et al. 2020; Wang et al. 2021b; Yang et al. 2020b). That indicated that ferroptosis-related lncRNAs might serve as novel disease molecular biomarkers and therapeutic targets for the treatment of cancer. However, the possible role of the ferroptosis-related lncRNA signature as a potentially useful tactics of treatment have not been reported in HCC. Therefore, we developed a FRlncRNAs signature with great prognosis and predictive value. Meanwhile, we also explored its effect in the response to ICB therapy for the patients with HCC.

Currently, the ferroptosis-related lncRNA signature has only been explored in head and neck 
279

280

281

282

283

284

285

286

287

288

289

290

291

292

293

294

295

296

297

298

299

300

301

302

303

304

305

306

307

308

309

310

311

312

313

314

squamous cell carcinoma (Tang et al. 2021). However, that study had several limitations, such as: small clinical sample sizes and lack of independent external validation datasets, which might lead to unreliable results. Furthermore, other confounding factors like comorbidities or alcohol consumption may also affect the robustness and accuracy of signature, which contained hemochromatosis, alcoholic liver disease, and nonalcoholic fatty liver disease, etc (Mao et al. 2020). Considering the above problems, the training cohort and independent validation cohort were utilized to develop a novel FRIncRNAs signature, which could predict the survival of patients with HCC. The results showed that the FRIncRNAs signature mainly involved DNA repair, glycolysis, MYC targets, and tumor-related signaling pathways. Those patients in the high-risk group were associated with worse OS. In addition, this FRIncRNAs signature was credible for predicting the prognosis of patients with $\mathrm{HCC}$, with a high AUC value (average AUC $>0.65$ ), and might serve as an indicator to measure the response of patients with HCC to ICB immunotherapy.

With the development of immune checkpoint inhibitors, ICB immunotherapy, as emerging strategies, has revealed treatment effects in HCC (Llovet et al. 2018; Pitt et al. 2016). Currently, immunotherapy has provided a novel promising treatment strategy for HCC (Sim \& Knox 2018). Unfortunately, more than two-third of patients did not respond to ICB treatment (Mushtaq et al. 2018). A new research demonstrated that ferroptosis combined with ICIs could synergistically enhance anti-tumor activity, even in ICI-resistant tumors (Tang et al. 2020). Thus, a novel FRIncRNAs signature was established to investigate the relationship between ICIs and ferroptosis, and predict ICB immunotherapy responses. In our study, the FRncRNAs signature was discovered to be associated with ICIs (i.e., PD-1, CTLA-4, and TIM-3), which indicated that the FRIncRNAs signature have potential to be used to measure the response to ICB therapy. At the same time, the expression levels of these ICIs in high-risk group were higher compared with low- group. That indicated that the FRIncRNAs signature could be applied to predict the expression level of ICIs and have the potential to guide ICB immunotherapy strategies. Moreover, the FRlncRNAs signature was connected with TICIs (B cell, Macrophage, Myeloid dendritic cell, Neutrophil, and CD4+ T cell) in HCC, which implies that this signature may play an important role in immune infiltration. Notably, these findings were consistent with previous studies manifesting that several lncRNAs served as regulators in tumor immunity, for instance immune cell infiltration and antigen release (Carpenter \& Fitzgerald 2018; Denaro et al. 2019).

Previous studies have revealed that lncRNAs participated in different biological processes such as immune regulation (Denaro et al. 2019), DNA repair and cell cycle (Hu et al. 2018; Majidinia \& Yousefi 2016), and metabolism (Denaro et al. 2019), etc. Among this FRlncRNAs signature (RHPN1-AS1, MAPKAPK5-AS1, and PART1) of our study, RHPN1-AS1 could facilitate cell proliferation, and invasion via activating PI3K/AKT/mTOR pathway in HCC (Song et al. 2020). Another study revealed that RHPN1-AS1 promoted the progression of HCC through regulating 
315

316

317

318

319

320

321

322

323

324

325

326

327

328

329

330

331

332

333

miR-596/IGF2BP2 axis (Fen et al. 2020). MAPKAPK5-AS1/PLAGL2/HIF-1 $\alpha$ signaling pathway was found to drive the progression of HCC and MAPKAPK5-AS1 might be a novel therapeutic target (Wang et al. 2021a). Furthermore, MAPKAPK5-AS1 has been discovered to promote the progression of colorectal cancer and thyroid cancer (Ji et al. 2019; Yang et al. 2020a; Zhou et al. 2020b). PART1 was involved in cell migration and invasion, and it could facilitate progression of HCC (Pu et al. 2020; Zhou et al. 2020a). PART1 also played a vital role in the occurrence and development of other cancers. Downregulated PART1 could suppress proliferation and accelerate apoptosis in bladder cancer (Hu et al. 2019). PART1 was regarded as a novel target in the treatment of prostate cancer (Sun et al. 2018). A study found that PART1 could promote cell proliferation in non-small-cell lung cancer cells by targeting miR-17-5p (Chen et al. 2021). Above evidences revealed that these three FRlncRNAs played important roles in development and prognosis of HCC. However, there is no research on their role in the prognosis of HCC via ferroptosis-related mechanism. Our findings may provide a new perspective for the treatment of HCC through ferroptosis-induction in the future.

Additionally, our results showed that the new FRlncRNAs signature possessed a highly predictive ability for OS prediction in the patients with HCC. Stratification analysis indicated that the FRlncRNAs signature based on risk grouping still possessed great predictive ability for survival prediction in each stratum of age ( $<65$ or $>=65$ patients), stage (Stage $1-2$ or Stage $3-4$ patients), grade (Grade 1-2 or Grade 3-4), and male patients, etc.

Several issues remained in the current study. First, the clinical sample size was not large. Second, the prognostic model was demanded to be validated in other enormous datasets to guarantee its robustness. Third, study has shown that HCC could be resistant to conventional chemotherapeutic, which might be associated with induction of ferroptosis-resistance (Galmiche 2019). However, due to the lack of patients of chemotherapy or radiotherapy in this study, it is impossible to confirm whether the signature can be applied to predict resistance to classical chemotherapy or radiotherapy through ferroptosis-induction. Fourth, the functional experiments should be implemented to reveal the potential biological mechanisms for predicting the influence of FRlncRNAs.

\section{CONCLUSIONS}

In conclusion, we identified a novel FRncRNAs signature related to prognosis of the patients with HCC, which could be utilized as a powerful tool in predicting the prognosis of patients with HCC. The FRlncRNAs signature could divide clinical characteristic subgroups according to survival. In addition, the signature was connected with ICB targets and TICIs. Hence, our study afforded a possible strategy for individualized risk stratification of the patients with HCC and 
350

351

352

353

354

355

356

357

358

359

360

361

362

363

364

365

366

367

368

369

370

371

372

373

374

375

376

377

378

379

380

381

382

383

384

evaluation response to ICB immunotherapy. The three FRncRNAs might be potential therapeutic targets of HCC.

\section{ADDITIONAL INFORMATION AND DECLARATIONS}

\section{Abbreviations}

HCC: Hepatocellular carcinoma; lncRNA: Long non-coding RNA; GO: Gene Ontology; KEGG: Kyoto Encyclopedia of Genes and Genomes; FRlncRNAs: ferroptosis-related lncRNAs; ROC: receiver operating characteristic; OS: overall survival; KM: Kaplan-Meier; GSEA: Gene set enrichment analyses; ssGSEA: single-sample gene set enrichment analysis (ssGSEA); TIICs: tumor-infiltrating immune cells; ICB: immune checkpoint blockade; FDR: false discovery rate. NAFLD: nonalcoholic fatty liver disease; HBV: hepatitis B virus; $\mathrm{HCV}$ : hepatitis $\mathrm{C}$ virus.

\section{Acknowledgements}

Not applicable.

\section{Funding}

This study was funded by the project of the Natural Science Foundation of Guangdong Province(2018A030313632), the Innovation and Entrepreneurship Training Program for College Students of GZUCM (S201910572049, 201910572168) and GZUCM Science Fund for Creative Research Groups (2016KYTD10) to Jin Zhang. The project of Traditional Chinese Medicine Bureau of Guangdong Province (20211118) to Fuping Ding. The Innovation and Entrepreneurship Training Program for College Students of GZUCM (201910572193) to Jin Huang.

\section{Availability of data and materials}

The following information was provided according to data availability:

Data is available at NCBI GEO under accession number GSE14520. All analyzed or generated data are included in the published manuscript and its supplementary document.

\section{Ethics approval and consent to participate}

Since the data involved in this study are from public databases (TCGA and GEO databases) and strictly follow their publication guidelines, there is no need for approval by the ethics committee.

\section{Competing Interests}

The authors declare there are no competing interests. 
385

386

387

388

389

390

391

392

393

394

395

396

397

398

399

400

401

402

403

404

405

406

407

408

409

410

411

412

413

414

415

416

417

418

419

420

421

422

\section{Consent for publication}

Not applicable.

\section{Supplementary Material}

Supplementary tables.

\section{Author Contributions}

J.Y.L. and Y.F.Z. contributed equally to this study. J.Y.L., Y.F.Z., and W.G.Z. conceived and designed the experiments; J.Y.L., Y.F.Z., W.G.Z., and W.H.D. composed or reviewed drafts of the paper; J.Y.L., and W.G.Z. analyzed and interpreted the data; Z.Y.C. completed the arrangement of figures and tables; All authors read and approved the final draft.

\section{REFERENCES}

Ai W, Li F, Yu HH, Liang ZH, and Zhao HP. 2020. Up-regulation of long noncoding RNA LINC00858 is associated with poor prognosis in gastric cancer. J Gene Med:e3179. 10.1002/jgm.3179

Bray F, Ferlay J, Soerjomataram I, Siegel RL, Torre LA, and Jemal A. 2018. Global cancer statistics 2018: GLOBOCAN estimates of incidence and mortality worldwide for 36 cancers in 185 countries. CA Cancer J Clin 68:394-424. 10.3322/caac.21492

Cancer Genome Atlas Research Network. Electronic address wbe, and Cancer Genome Atlas Research N. 2017. Comprehensive and Integrative Genomic Characterization of Hepatocellular Carcinoma. Cell 169:1327-1341 e1323. 10.1016/j.cell.2017.05.046

Carpenter S, and Fitzgerald KA. 2018. Cytokines and Long Noncoding RNAs. Cold Spring Harb Perspect Biol 10. 10.1101/cshperspect.a028589

Cech TR, and Steitz JA. 2014. The noncoding RNA revolution-trashing old rules to forge new ones. Cell 157:77-94. 10.1016/j.cell.2014.03.008

Charoentong P, Finotello F, Angelova M, Mayer C, Efremova M, Rieder D, Hackl H, and Trajanoski Z. 2017. Pan-cancer Immunogenomic Analyses Reveal GenotypeImmunophenotype Relationships and Predictors of Response to Checkpoint Blockade. Cell Rep 18:248-262. 10.1016/j.celrep.2016.12.019

Chen Y, Zhou X, Huang C, Li L, Qin Y, Tian Z, He J, and Liu H. 2021. LncRNA PART1 promotes cell proliferation and progression in non-small-cell lung cancer cells via sponging miR-175p. J Cell Biochem 122:315-325. 10.1002/jcb.29714

Denaro N, Merlano MC, and Lo Nigro C. 2019. Long noncoding RNAs as regulators of cancer immunity. Mol Oncol 13:61-73. 10.1002/1878-0261.12413

DiStefano JK. 2017. Long noncoding RNAs in the initiation, progression, and metastasis of hepatocellular carcinoma. Noncoding RNA Res 2:129-136. 10.1016/j.ncrna.2017.11.001

Dixon SJ, Lemberg KM, Lamprecht MR, Skouta R, Zaitsev EM, Gleason CE, Patel DN, Bauer 
AJ, Cantley AM, Yang WS, Morrison B, 3rd, and Stockwell BR. 2012. Ferroptosis: an iron-dependent form of nonapoptotic cell death. Cell 149:1060-1072. 10.1016/j.cell.2012.03.042

European Association for the Study of the Liver. Electronic address eee, and European Association for the Study of the L. 2018. EASL Clinical Practice Guidelines: Management of hepatocellular carcinoma. J Hepatol 69:182-236. 10.1016/j.jhep.2018.03.019

Fen H, Hongmin Z, Wei W, Chao Y, Yang Y, Bei L, and Zhihua S. 2020. RHPN1-AS1 Drives the Progression of Hepatocellular Carcinoma via Regulating miR-596/IGF2BP2 Axis. Curr Pharm Des 25:4630-4640. 10.2174/1381612825666191105104549

Finotello F, Mayer C, Plattner C, Laschober G, Rieder D, Hackl H, Krogsdam A, Loncova Z, Posch W, Wilflingseder D, Sopper S, Ijsselsteijn M, Brouwer TP, Johnson D, Xu Y, Wang Y, Sanders ME, Estrada MV, Ericsson-Gonzalez P, Charoentong P, Balko J, de Miranda N, and Trajanoski Z. 2019. Molecular and pharmacological modulators of the tumor immune contexture revealed by deconvolution of RNA-seq data. Genome Med 11:34. 10.1186/s 13073-019-0638-6

Frankel LB, Lubas M, and Lund AH. 2017. Emerging connections between RNA and autophagy. Autophagy 13:3-23. 10.1080/15548627.2016.1222992

Galmiche A. 2019. Ferroptosis in Liver Disease. In: Tang D, ed. Ferroptosis in Health and Disease. Cham: Springer International Publishing, 239-248.

Goodman A, Patel SP, and Kurzrock R. 2017. PD-1-PD-L1 immune-checkpoint blockade in Bcell lymphomas. Nat Rev Clin Oncol 14:203-220. 10.1038/nrclinonc.2016.168

Hassannia B, Vandenabeele P, and Vanden Berghe T. 2019. Targeting Ferroptosis to Iron Out Cancer. Cancer Cell 35:830-849. 10.1016/j.ccell.2019.04.002

He J, Zuo Q, Hu B, Jin H, Wang C, Cheng Z, Deng X, Yang C, Ruan H, Yu C, Zhao F, Yao M, Fang J, Gu J, Zhou J, Fan J, Qin W, Yang XR, and Wang H. 2019. A novel, liver-specific long noncoding RNA LINC01093 suppresses HCC progression by interaction with IGF2BP1 to facilitate decay of GLI1 mRNA. Cancer Lett 450:98-109. 10.1016/j.canlet.2019.02.033

Hu X, Feng H, Huang H, Gu W, Fang Q, Xie Y, Qin C, and Hu X. 2019. Downregulated Long Noncoding RNA PART1 Inhibits Proliferation and Promotes Apoptosis in Bladder Cancer. Technol Cancer Res Treat 18:1533033819846638. 10.1177/1533033819846638

Hu X, Sood AK, Dang CV, and Zhang L. 2018. The role of long noncoding RNAs in cancer: the dark matter matters. Curr Opin Genet Dev 48:8-15. 10.1016/j.gde.2017.10.004

Jennis M, Kung CP, Basu S, Budina-Kolomets A, Leu JI, Khaku S, Scott JP, Cai KQ, Campbell MR, Porter DK, Wang X, Bell DA, Li X, Garlick DS, Liu Q, Hollstein M, George DL, and Murphy ME. 2016. An African-specific polymorphism in the TP53 gene impairs p53 tumor suppressor function in a mouse model. Genes Dev 30:918-930. 10.1101/gad.275891.115

Ji H, Hui B, Wang J, Zhu Y, Tang L, Peng P, Wang T, Wang L, Xu S, Li J, and Wang K. 2019. Long noncoding RNA MAPKAPK5-AS1 promotes colorectal cancer proliferation by partly silencing p21 expression. Cancer Sci 110:72-85. 10.1111/cas.13838

Kim JE, Patel MA, Mangraviti A, Kim ES, Theodros D, Velarde E, Liu A, Sankey EW, Tam A, 
464

465

466

467

468

469

470

471

472

473

474

475

476

477

478

479

480

481

482

483

484

485

486

487

488

489

490

491

492

493

494

495

496

497

498

499

500

501

502

503

504

Xu H, Mathios D, Jackson CM, Harris-Bookman S, Garzon-Muvdi T, Sheu M, Martin AM, Tyler BM, Tran PT, Ye X, Olivi A, Taube JM, Burger PC, Drake CG, Brem H, Pardoll DM, and Lim M. 2017. Combination Therapy with Anti-PD-1, Anti-TIM-3, and Focal Radiation Results in Regression of Murine Gliomas. Clin Cancer Res 23:124-136. 10.1158/1078-0432.CCR-15-1535

Li CY, Zhang WW, Xiang JL, Wang XH, Wang JL, and Li J. 2019. Integrated analysis highlights multiple long noncoding RNAs and their potential roles in the progression of human esophageal squamous cell carcinoma. Oncol Rep 42:2583-2599. 10.3892/or.2019.7377

Li T, Fan J, Wang B, Traugh N, Chen Q, Liu JS, Li B, and Liu XS. 2017. TIMER: A Web Server for Comprehensive Analysis of Tumor-Infiltrating Immune Cells. Cancer Res 77:e108e110. 10.1158/0008-5472.CAN-17-0307

Liang C, Zhang X, Yang M, and Dong X. 2019. Recent Progress in Ferroptosis Inducers for Cancer Therapy. Adv Mater 31:e1904197. 10.1002/adma.201904197

Llovet JM, Montal R, Sia D, and Finn RS. 2018. Molecular therapies and precision medicine for hepatocellular carcinoma. Nat Rev Clin Oncol 15:599-616. 10.1038/s41571-018-0073-4

Llovet JM, Zucman-Rossi J, Pikarsky E, Sangro B, Schwartz M, Sherman M, and Gores G. 2016. Hepatocellular carcinoma. Nat Rev Dis Primers 2:16018. 10.1038/nrdp.2016.18

Louandre C, Marcq I, Bouhlal H, Lachaier E, Godin C, Saidak Z, Francois C, Chatelain D, Debuysscher V, Barbare JC, Chauffert B, and Galmiche A. 2015. The retinoblastoma (Rb) protein regulates ferroptosis induced by sorafenib in human hepatocellular carcinoma cells. Cancer Lett 356:971-977. 10.1016/j.canlet.2014.11.014

Lu J, Xu F, and Lu H. 2020. LncRNA PVT1 regulates ferroptosis through miR-214-mediated TFR1 and p53. Life Sci 260:118305. 10.1016/j.1fs.2020.118305

Majidinia M, and Yousefi B. 2016. Long non-coding RNAs in cancer drug resistance development. DNA Repair (Amst) 45:25-33. 10.1016/j.dnarep.2016.06.003

Mao L, Zhao T, Song Y, Lin L, Fan X, Cui B, Feng H, Wang X, Yu Q, Zhang J, Jiang K, Wang B, and Sun C. 2020. The emerging role of ferroptosis in non-cancer liver diseases: hype or increasing hope? Cell Death Dis 11:518. 10.1038/s41419-020-2732-5

Mathy NW, and Chen XM. 2017. Long non-coding RNAs (lncRNAs) and their transcriptional control of inflammatory responses. $J$ Biol Chem 292:12375-12382. 10.1074/jbc.R116.760884

Mushtaq MU, Papadas A, Pagenkopf A, Flietner E, Morrow Z, Chaudhary SG, and Asimakopoulos F. 2018. Tumor matrix remodeling and novel immunotherapies: the promise of matrix-derived immune biomarkers. $J$ Immunother Cancer 6:65. 10.1186/s40425-018-0376-0

Newman AM, Liu CL, Green MR, Gentles AJ, Feng W, Xu Y, Hoang CD, Diehn M, and Alizadeh AA. 2015. Robust enumeration of cell subsets from tissue expression profiles. Nat Methods 12:453-457. 10.1038/nmeth.3337

Nishino M, Ramaiya NH, Hatabu H, and Hodi FS. 2017. Monitoring immune-checkpoint blockade: response evaluation and biomarker development. Nat Rev Clin Oncol 14:655668. 10.1038/nrclinonc. 2017.88 
505

506

507

508

509

510

511

512

513

514

515

516

517

518

519

520

521

522

523

524

525

526

527

528

529

530

531

532

533

534

535

536

537

538

539

540

541

542

543

544

545

Oweira H, Petrausch U, Helbling D, Schmidt J, Mehrabi A, Schob O, Giryes A, and AbdelRahman O. 2017. Prognostic value of site-specific extra-hepatic disease in hepatocellular carcinoma: a SEER database analysis. Expert Rev Gastroenterol Hepatol 11:695-701. 10.1080/17474124.2017.1294485

Pitt JM, Vetizou M, Daillere R, Roberti MP, Yamazaki T, Routy B, Lepage P, Boneca IG, Chamaillard M, Kroemer G, and Zitvogel L. 2016. Resistance Mechanisms to ImmuneCheckpoint Blockade in Cancer: Tumor-Intrinsic and -Extrinsic Factors. Immunity 44:1255-1269. 10.1016/j.immuni.2016.06.001

Pu J, Tan C, Shao Z, Wu X, Zhang Y, Xu Z, Wang J, Tang Q, and Wei H. 2020. Long Noncoding RNA PART1 Promotes Hepatocellular Carcinoma Progression via Targeting miR-5903p/HMGB2 Axis. Onco Targets Ther 13:9203-9211. 10.2147/OTT.S259962

Racle J, de Jonge K, Baumgaertner P, Speiser DE, and Gfeller D. 2017. Simultaneous enumeration of cancer and immune cell types from bulk tumor gene expression data. Elife 6. 10.7554/eLife. 26476

Sangro B, Sarobe P, Hervas-Stubbs S, and Melero I. 2021. Advances in immunotherapy for hepatocellular carcinoma. Nat Rev Gastroenterol Hepatol. 10.1038/s41575-021-00438-0

Schulze K, Nault JC, and Villanueva A. 2016. Genetic profiling of hepatocellular carcinoma using next-generation sequencing. J Hepatol 65:1031-1042. 10.1016/j.jhep.2016.05.035

Shi J, Jiang D, Yang S, Zhang X, Wang J, Liu Y, Sun Y, Lu Y, and Yang K. 2020. LPAR1, Correlated With Immune Infiltrates, Is a Potential Prognostic Biomarker in Prostate Cancer. Front Oncol 10:846. 10.3389/fonc.2020.00846

Sim HW, and Knox J. 2018. Hepatocellular carcinoma in the era of immunotherapy. Curr Probl Cancer 42:40-48. 10.1016/j.currproblcancer.2017.10.007

Song XZ, Ren XN, Xu XJ, Ruan XX, Wang YL, and Yao TT. 2020. LncRNA RHPN1-AS1 Promotes Cell Proliferation, Migration and Invasion Through Targeting miR-7-5p and Activating PI3K/AKT/mTOR Pathway in Hepatocellular Carcinoma. Technol Cancer Res Treat 19:1533033820957023. 10.1177/1533033820957023

Stockwell BR, Friedmann Angeli JP, Bayir H, Bush AI, Conrad M, Dixon SJ, Fulda S, Gascon S, Hatzios SK, Kagan VE, Noel K, Jiang X, Linkermann A, Murphy ME, Overholtzer M, Oyagi A, Pagnussat GC, Park J, Ran Q, Rosenfeld CS, Salnikow K, Tang D, Torti FM, Torti SV, Toyokuni S, Woerpel KA, and Zhang DD. 2017. Ferroptosis: A Regulated Cell Death Nexus Linking Metabolism, Redox Biology, and Disease. Cell 171:273-285. 10.1016/j.cell.2017.09.021

Sun M, Geng D, Li S, Chen Z, and Zhao W. 2018. LncRNA PART1 modulates toll-like receptor pathways to influence cell proliferation and apoptosis in prostate cancer cells. Biol Chem 399:387-395. 10.1515/hsz-2017-0255

Sun X, Niu X, Chen R, He W, Chen D, Kang R, and Tang D. 2016a. Metallothionein-1G facilitates sorafenib resistance through inhibition of ferroptosis. Hepatology 64:488-500. 10.1002/hep.28574

Sun X, Ou Z, Chen R, Niu X, Chen D, Kang R, and Tang D. 2016b. Activation of the p62-Keap1NRF2 pathway protects against ferroptosis in hepatocellular carcinoma cells. Hepatology

Peer] reviewing PDF | (2021:03:59351:1:1:NEW 20 May 2021) 
546

547

548

549

550

551

552

553

554

555

556

557

558

559

560

561

562

563

564

565

566

567

568

569

570

571

572

573

574

575

576

577

578

579

580

581

582

583

584

585

586

63:173-184. 10.1002/hep.28251

Tang R, Xu J, Zhang B, Liu J, Liang C, Hua J, Meng Q, Yu X, and Shi S. 2020. Ferroptosis, necroptosis, and pyroptosis in anticancer immunity. J Hematol Oncol 13:110. 10.1186/s 13045-020-00946-7

Tang Y, Li C, Zhang Y-J, and Wu Z-H. 2021. Ferroptosis-Related Long Non-Coding RNA signature predicts the prognosis of Head and neck squamous cell carcinoma. International Journal of Biological Sciences 17:702-711. 10.7150/ijbs.55552

Wang H, Liang L, Dong Q, Huan L, He J, Li B, Yang C, Jin H, Wei L, Yu C, Zhao F, Li J, Yao M, Qin W, Qin L, and He X. 2018. Long noncoding RNA miR503HG, a prognostic indicator, inhibits tumor metastasis by regulating the HNRNPA2B1/NF-kappaB pathway in hepatocellular carcinoma. Theranostics 8:2814-2829. 10.7150/thno.23012

Wang L, Sun L, Liu R, Mo H, Niu Y, Chen T, Wang Y, Han S, Tu K, and Liu Q. 2021a. Long non-coding RNA MAPKAPK5-AS1/PLAGL2/HIF-1alpha signaling loop promotes hepatocellular carcinoma progression. J Exp Clin Cancer Res 40:72. 10.1186/s13046-02101868-z

Wang Z, Chen X, Liu N, Shi Y, Liu Y, Ouyang L, Tam S, Xiao D, Liu S, Wen F, and Tao Y. 2021b. A Nuclear Long Non-Coding RNA LINC00618 Accelerates Ferroptosis in a Manner Dependent upon Apoptosis. Mol Ther 29:263-274. 10.1016/j.ymthe.2020.09.024

Wei L, Wang X, Lv L, Liu J, Xing H, Song Y, Xie M, Lei T, Zhang N, and Yang M. 2019. The emerging role of microRNAs and long noncoding RNAs in drug resistance of hepatocellular carcinoma. Mol Cancer 18:147. 10.1186/s12943-019-1086-z

Wing-Sum Cheu J, and Chak-Lui Wong C. 2021. Mechanistic Rationales guiding Combination Hepatocellular Carcinoma Therapies involving Immune Checkpoint Inhibitors. Hepatology. 10.1002/hep.31840

Yang JD, Hainaut P, Gores GJ, Amadou A, Plymoth A, and Roberts LR. 2019. A global view of hepatocellular carcinoma: trends, risk, prevention and management. Nat Rev Gastroenterol Hepatol 16:589-604. 10.1038/s41575-019-0186-y

Yang T, Chen WC, Shi PC, Liu MR, Jiang T, Song H, Wang JQ, Fan RZ, Pei DS, and Song J. 2020a. Long noncoding RNA MAPKAPK5-AS1 promotes colorectal cancer progression by cis-regulating the nearby gene MK5 and acting as a let-7f-1-3p sponge. $J$ Exp Clin Cancer Res 39:139. 10.1186/s13046-020-01633-8

Yang Y, Tai W, Lu N, Li T, Liu Y, Wu W, Li Z, Pu L, Zhao X, Zhang T, and Dong Z. 2020 b. lncRNA ZFAS1 promotes lung fibroblast-to-myofibroblast transition and ferroptosis via functioning as a ceRNA through miR-150-5p/SLC38A1 axis. Aging (Albany NY) 12:90859102. 10.18632/aging.103176

Ye J, Zhang J, Lv Y, Wei J, Shen X, Huang J, Wu S, and Luo X. 2019. Integrated analysis of a competing endogenous RNA network reveals key long noncoding RNAs as potential prognostic biomarkers for hepatocellular carcinoma. J Cell Biochem 120:13810-13825. 10.1002/jcb.28655

Yi M, Nissley DV, McCormick F, and Stephens RM. 2020. ssGSEA score-based Ras dependency indexes derived from gene expression data reveal potential Ras addiction mechanisms with 
587

588

589

590

591

592

593

594

595

596

597

598

599

600

601

602

603

604

605

606

607

608

609

610

611

612

613

614

615

616

617

618

619

620

621

622

623

624

625

possible clinical implications. Sci Rep 10:10258. 10.1038/s41598-020-66986-8

Yoshihara K, Shahmoradgoli M, Martinez E, Vegesna R, Kim H, Torres-Garcia W, Trevino V, Shen H, Laird PW, Levine DA, Carter SL, Getz G, Stemke-Hale K, Mills GB, and Verhaak RG. 2013. Inferring tumour purity and stromal and immune cell admixture from expression data. Nat Commun 4:2612. 10.1038/ncomms3612

Yuan H, Li X, Zhang X, Kang R, and Tang D. 2016. CISD1 inhibits ferroptosis by protection against mitochondrial lipid peroxidation. Biochem Biophys Res Commun 478:838-844. 10.1016/j.bbrc.2016.08.034

Zeng J, Liu Z, Zhang C, Hong T, Zeng F, Guan J, Tang S, and Hu Z. 2020. Prognostic value of long non-coding RNA SNHG20 in cancer: A meta-analysis. Medicine (Baltimore) 99:e19204. 10.1097/MD.0000000000019204

Zhai L, Ladomersky E, Lenzen A, Nguyen B, Patel R, Lauing KL, Wu M, and Wainwright DA. 2018. IDO1 in cancer: a Gemini of immune checkpoints. Cell Mol Immunol 15:447-457. 10.1038/cmi.2017.143

Zhao X, Liu Y, and Yu S. 2017. Long noncoding RNA AWPPH promotes hepatocellular carcinoma progression through YBX1 and serves as a prognostic biomarker. Biochim Biophys Acta Mol Basis Dis 1863:1805-1816. 10.1016/j.bbadis.2017.04.014

Zhou C, Wang P, Tu M, Huang Y, Xiong F, and Wu Y. 2020a. Long Non-Coding RNA PART1 Promotes Proliferation, Migration and Invasion of Hepatocellular Carcinoma Cells via miR-149-5p/MAP2K1 Axis. Cancer Manag Res 12:3771-3782. 10.2147/CMAR.S246311

Zhou N, and Bao J. 2020. FerrDb: a manually curated resource for regulators and markers of ferroptosis and ferroptosis-disease associations. Database (Oxford) 2020. 10.1093/database/baaa021

Zhou Y, Liu S, Luo Y, Zhang M, Jiang X, and Xiong Y. 2020b. IncRNA MAPKAPK5-AS1 promotes proliferation and migration of thyroid cancer cell lines by targeting miR-519e5p/YWHAH. Eur J Histochem 64. 10.4081/ejh.2020.3177

Zongyi Y, and Xiaowu L. 2020. Immunotherapy for hepatocellular carcinoma. Cancer Lett 470:817. $10.1016 /$ j.canlet.2019.12.002

\section{Figure and Table legend}

Figure. 1 The differentially expressed ferroptosis-related genes. (A) The Volcano plot of the differentially expressed ferroptosis-related genes. The red dots implied up-regulated and blue for down-regulated. The top 20 gene symbols with high variation were displayed in the plot. (B) The heatmap of 20 ferroptosis-related genes with high variation in training cohort. (C) The heatmap of 20 ferroptosis-related genes with high variation (except for TUBE1, and FANCD2) between tumor and normal samples in validation cohort. (D) The GO circle plot of functional enrichment. The red dots indicated up-regulation, while the blue indicated down-regulation. (E) The KEGG circle plot of enrichment analysis. The Z-score was directly proportional to the level of enrichment. 
627 Figure. 2 Identification of prognostic Ferroptosis-related IncRNAs (FRIncRNAs). (A) Forest 628 plots revealed prognosis-related FRlncRNAs based on the results of univariate Cox regression. (B) 629 ROC curve for FRlncRNAs signature at 1 year in training cohort. The cut-off score was 1.038 and 630 it was utilized to classify patients into high- or low-risk groups. (C) The relational network between signature and corresponding co-expression ferroptosis-related genes. (D) Sankey diagram indicated that the association between prognostic FRIncRNAs, ferroptosis-related genes, and risk type.

Figure. 3 The FRIncRNAs signature based on training cohort. (A) Kaplan-Meier (KM) curve for overall survival (OS) of patients with HCC in high- and low-risk group in training cohort. (B) The AUC values of the risk score and additional clinical characteristics. (C) Risk survival status plot in patients with HCC. (D) ROC curves at 1, 3, 5-year were applied to verify prognostic performance of FRlncRNAs signature established by training cohort.

Figure. 4 The FRIncRNAs signature was an independent prognostic indicator and possessed potential clinical value in training cohort. Univariate (A) and multivariate (B) Cox regression analysis of the FRlncRNAs signature in predicting patient survival. (C) A nomogram among clinical features (including the risk score) and OS of patients. (D-F) Calibration for assessing the consistence between the predicted and the actual OS at 1,3,5 years.

Figure. 5 The survival curves of the FRIncRNAs signature stratified by age, grade, stage, gender, etiology, radiotherapy, and family history. (A) $\geq 65$ years, (B) < 65 years, (C) grade 12, (D) grade 3-4, (E) stage 1-2, (F) stage 3-4, (G) Alcoholic liver disease, (H) NAFLD, (I) no family history, (J) male, (K) HBV positive, (L) HBV and HCV negative, (M) no radiotherapy patients.

Figure. 6 The verification of prognostic FRIncRNAs signature in validation cohort. (A) The KM curves of validation cohort revealed that high-risk group had statistical differences on OS period compared with low-risk group $(P<0.05)$. Univariate $(\mathrm{B})$ and multivariate $(\mathrm{C}) \mathrm{COX}$ regression for the FRlncRNAs signature established by training cohort. (D) AUC of ROC curves validated the predicted performance of signature in validation cohort.

Figure 7 Some cancer-related hallmarks and immunologic features regulated through the FRIncRNAs signature. (A) Cancer hallmarks. (B) Immunologic signatures. 
662

663 Figure. 8 The relationship between FRIncRNAs signature and TIICs, ICB molecules based on TIMER results. (A) Spearman correlation between the signature and B cell; (B) Spearman correlation between the signature and Macrophage cell. (C) Spearman correlation between the signature and Myeloid dendritic cell. (D) Spearman correlation between the signature and Neutrophil cell. (E) Spearman correlation between the signature and CD4+ T cell. (F) Spearman correlation between the signature and CD8+ T cell. (G-I) Significant positive association between our FRlncRNAs signature and ICB receptors PD-1 $(\mathrm{R}=0.17$; $\mathrm{P}=0.0019)$, CTLA-4 $(\mathrm{R}=0.19 ; \mathrm{P}<$ $0.001)$, and TIM-3 ( $\mathrm{R}=0.16 ; \mathrm{P}<0.001)$. (J-L) The comparison of the expression levels of PD-1, CTLA-4, and TIM-3 between high-risk and low- groups.

Figure. 9 Based on CIBERSORT, EPIC, ESTIMATE, MCP counter, QUANTISEQ, ssGSEA and TIMER algorithms, heatmap of immune infiltration in high- and low-risk group.

Figure. S1 The signature was irrelevant to IDO1, PD-L1, and PD-L2 in TCGA cohort.

Table 1 Clinical characteristics of the patients with HCC from TCGA cohort in this study.

Table 2 Univariate and multivariate independent prognostic analysis of FRlncRNAs signature in predicting patient survival.

Table S1 The source file of clinical information for patients with HCC.

Table S2 A list of ferroptosis-related genes from FerrDb Database.

Table S3 The differentially expressed ferroptosis-related genes.

Table S4 The GO and KEGG enrichment analysis based on differentially expressed ferroptosisrelated genes.

Table S5 Correlation analysis between differentially expressed ferroptosis-related genes and lncRNAs.

Table S6 Cox regression for screening FRlncRNAs associated with prognosis. 


\section{Figure 1}

The differentially expressed ferroptosis-related genes.

(A) The Volcano plot of the differentially expressed ferroptosis-related genes. The red dots implied up-regulated and blue for down-regulated. The top 20 gene symbols with high variation were displayed in the plot. (B) The heatmap of 20 ferroptosis-related genes with high variation in training cohort. (C) The heatmap of 20 ferroptosis-related genes with high variation (except for TUBE1, and FANCD2) between tumor and normal samples in validation cohort. (D) The GO circle plot of functional enrichment. The red dots indicated up-regulation, while the blue indicated down-regulation. (E) The KEGG circle plot of enrichment analysis. The Z-score was directly proportional to the level of enrichment. 
A

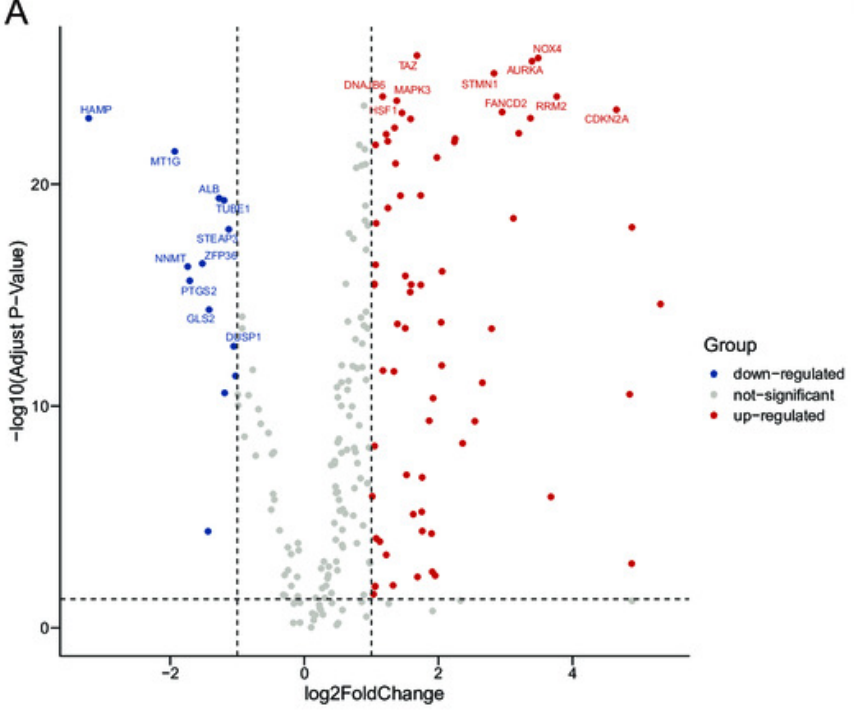

D
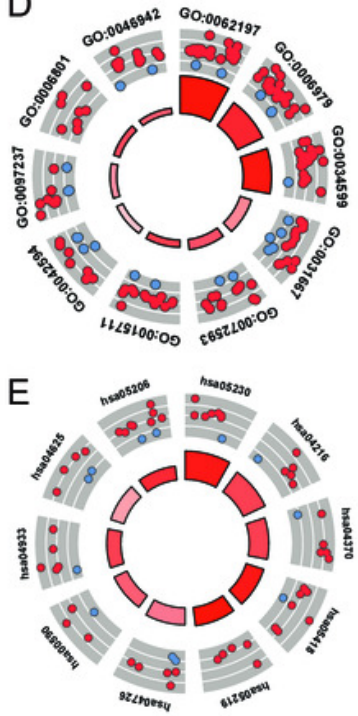

B

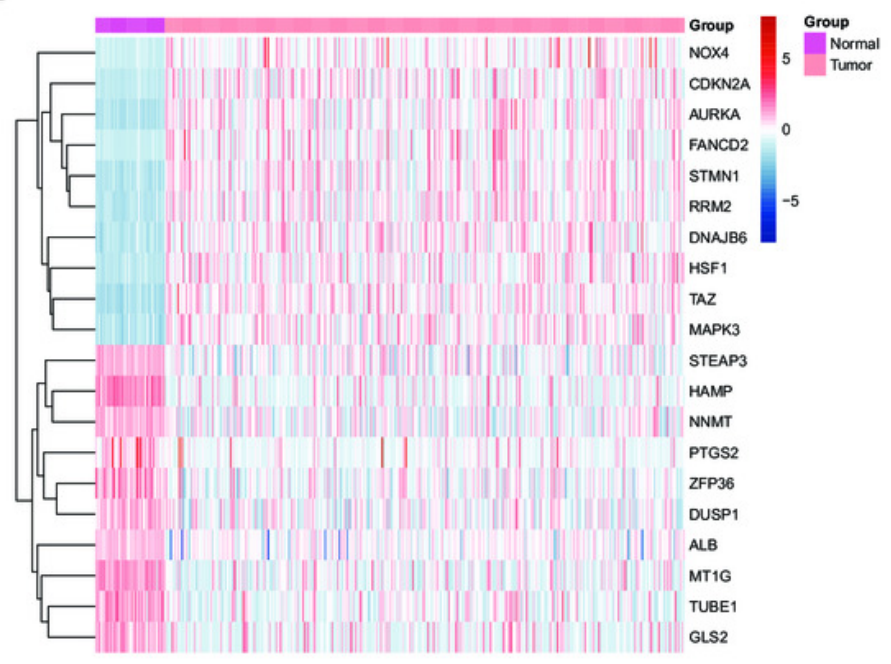

C

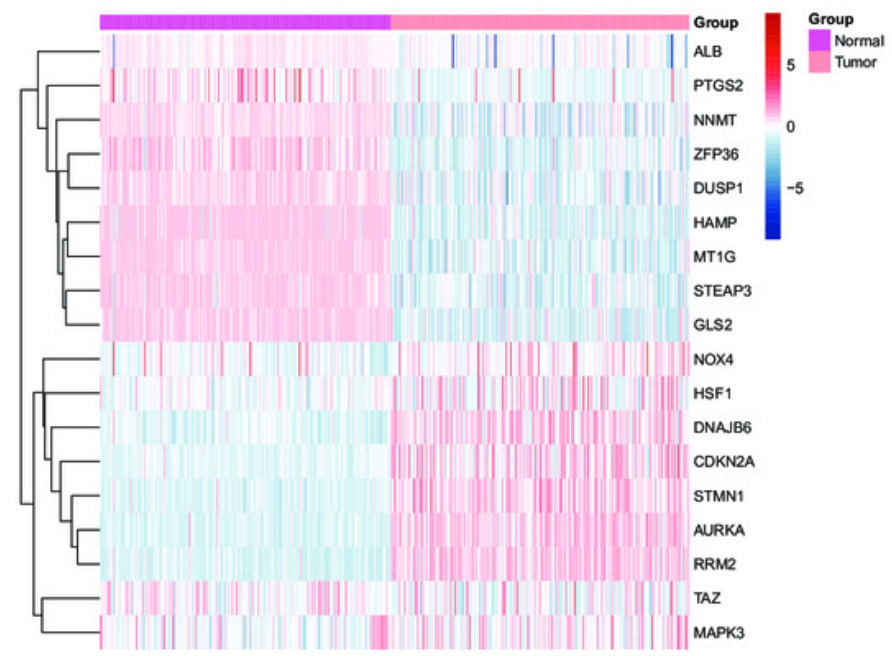




\section{Figure 2}

Identification of prognostic Ferroptosis-related IncRNAs (FRIncRNAs).

(A) Forest plots revealed prognosis-related FRIncRNAs based on the results of univariate Cox regression. (B) ROC curve for FRIncRNAs signature at 1 year in training cohort. The cut-off score was 1.038 and it was utilized to classify patients into high-or low-risk groups. (C) The relational network between signature and corresponding co-expression ferroptosis-related genes. (D) Sankey diagram indicated that the association between prognostic FRIncRNAs, ferroptosis-related genes, and risk type. 

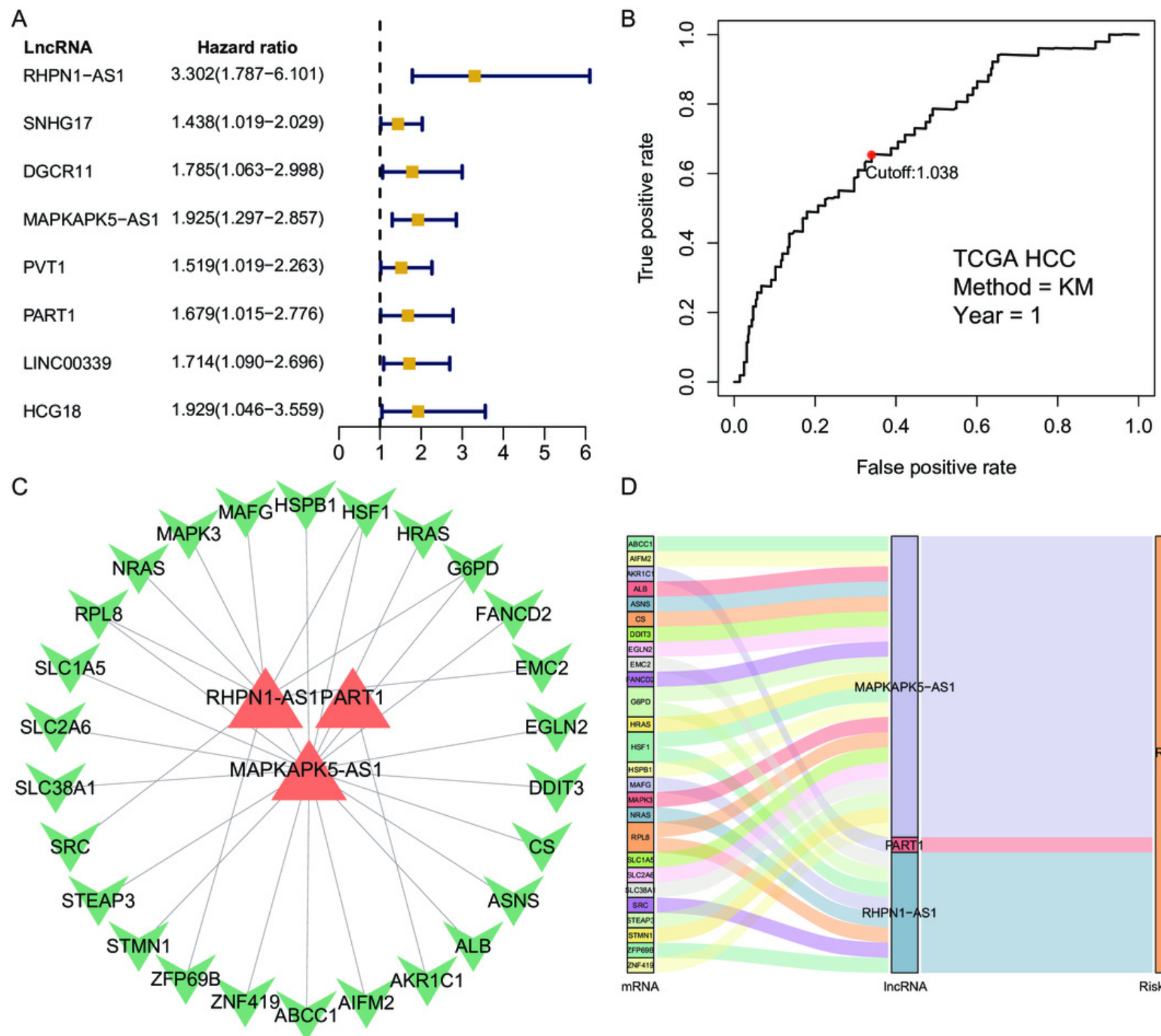

D

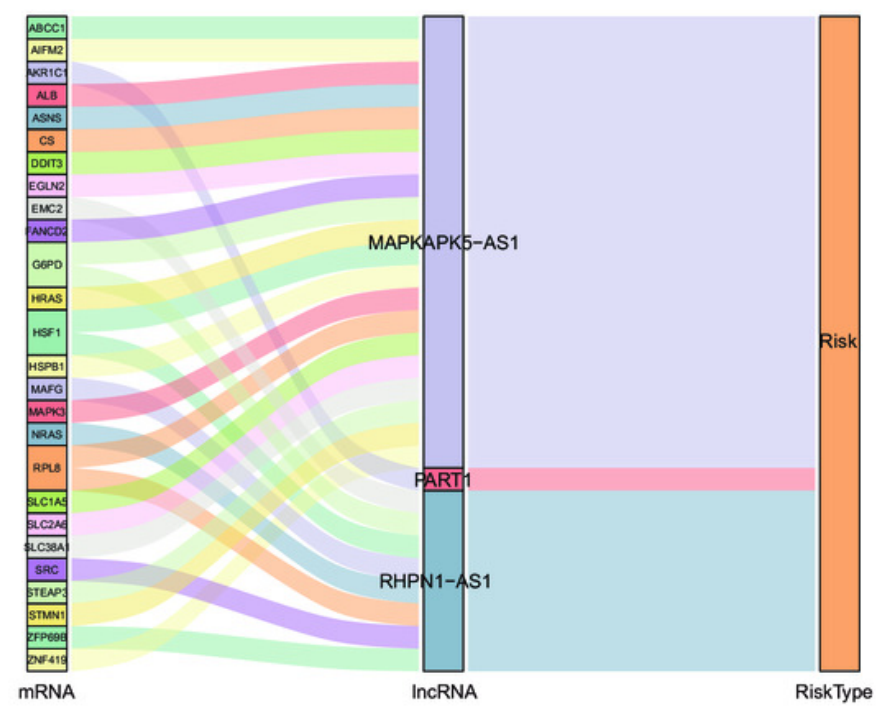


Figure 3

The FRIncRNAs signature based on training cohort.

(A) Kaplan-Meier (KM) curve for overall survival (OS) of patients with HCC in high- and lowrisk group in training cohort. (B) The AUC values of the risk score and additional clinical characteristics. (C) Risk survival status plot in patients with HCC. (D) ROC curves at 1, 3, 5year were applied to verify prognostic performance of FRIncRNAs signature established by training cohort. 
A
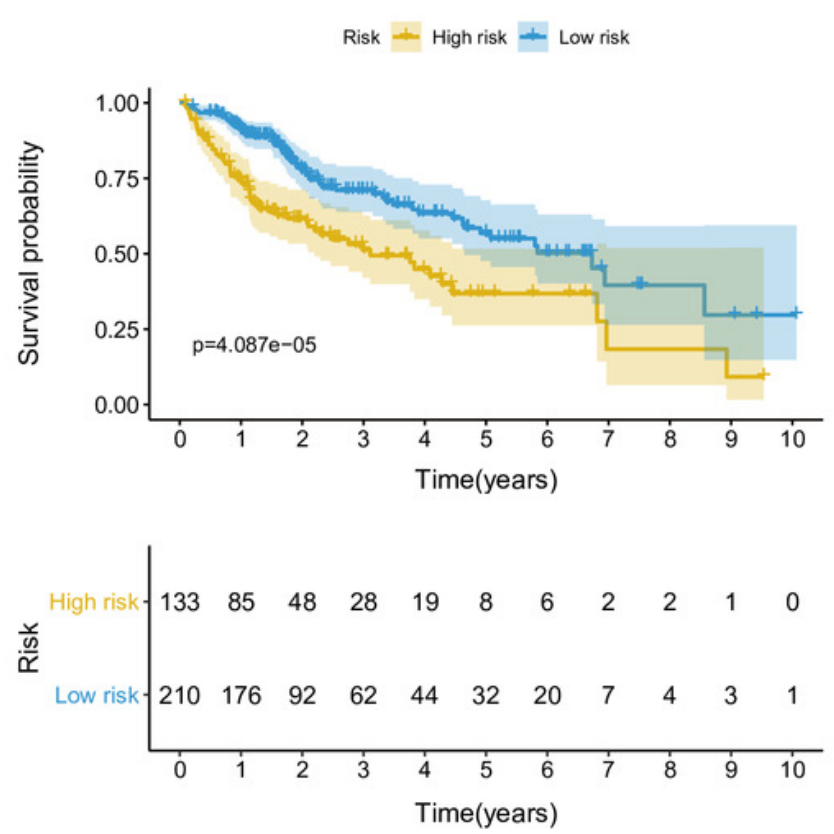

B

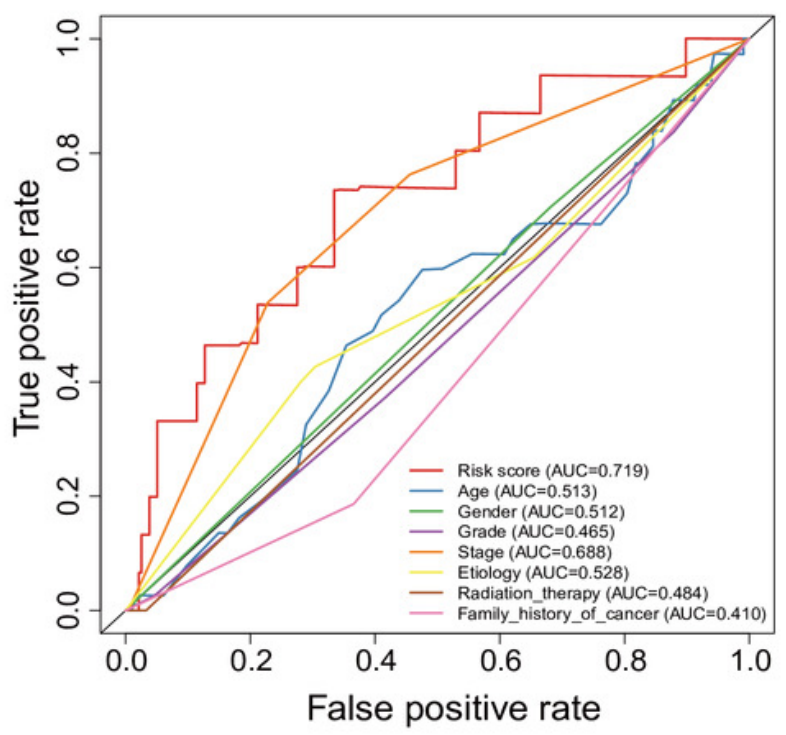

C
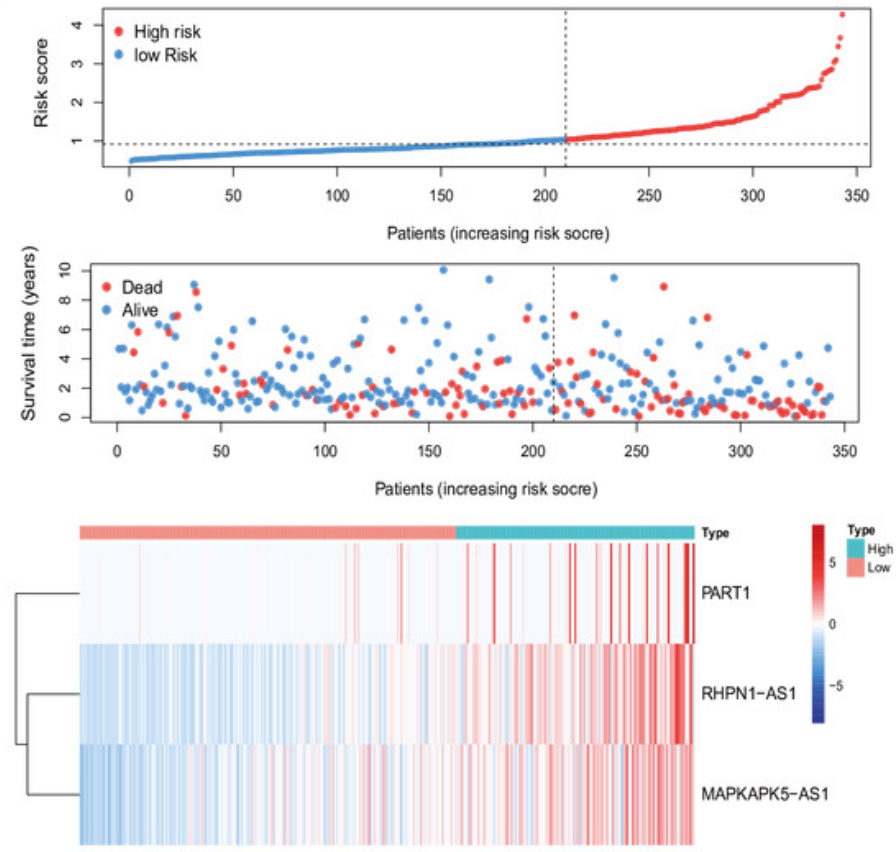

D

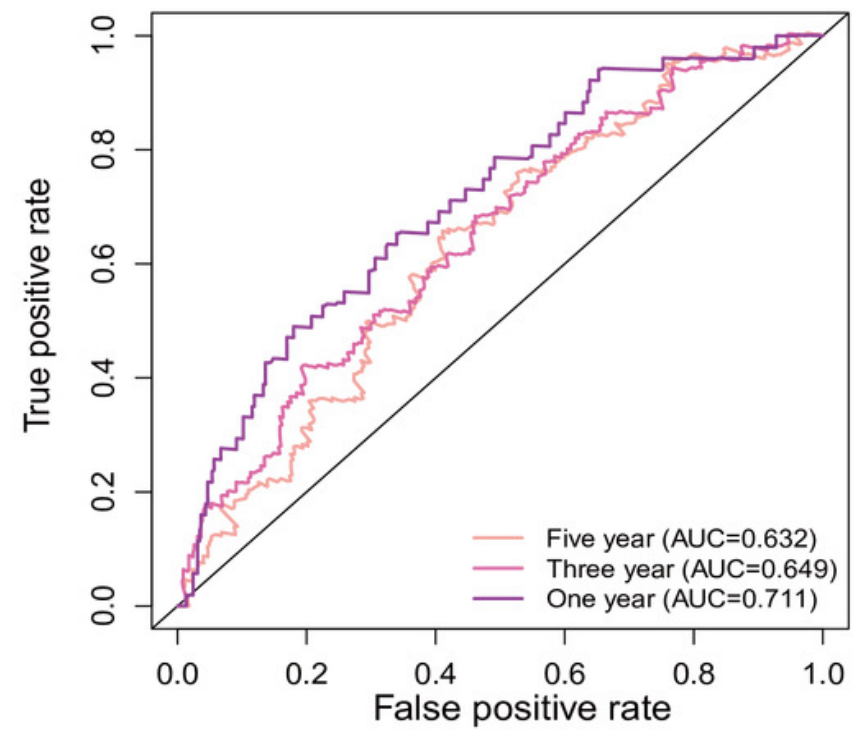




\section{Figure 4}

The FRIncRNAs signature was an independent prognostic indicator and possessed potential clinical value in training cohort.

Univariate $(A)$ and multivariate $(B)$ Cox regression analysis of the FRIncRNAs signature in predicting patient survival. (C) A nomogram among clinical features (including the risk score) and OS of patients. (D-F) Calibration for assessing the consistence between the predicted and the actual OS at 1, 3, 5 years. 
A

$\begin{array}{lcc}\text { Feature } & \text { P value } & \text { Hazard Ratio } \\ \text { Age } & 0.104 & 1.014(0.997-1.032) \\ \text { Gender } & 0.103 & 0.701(0.457-1.075) \\ \text { Grade } & 0.815 & 1.035(0.778-1.376) \\ \text { Stage } & <0.001 & 1.853(1.464-2.345) \\ \text { Etiology } & 0.158 & 1.135(0.952-1.352) \\ \text { Radiotherapy } & 0.815 & 1.147(0.362-3.636) \\ \text { Family_history } & 0.613 & 1.117(0.728-1.713) \\ \text { RiskScore } & <0.001 & 1.732(1.324-2.267)\end{array}$

C

Points

Age

Gender

Grade

Stage

RiskScore

Total Points
B

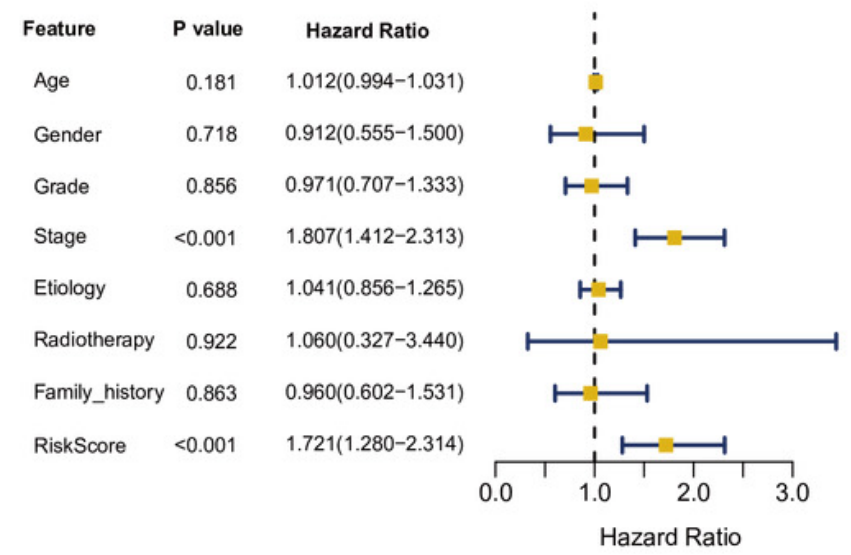

Hazard Ratio
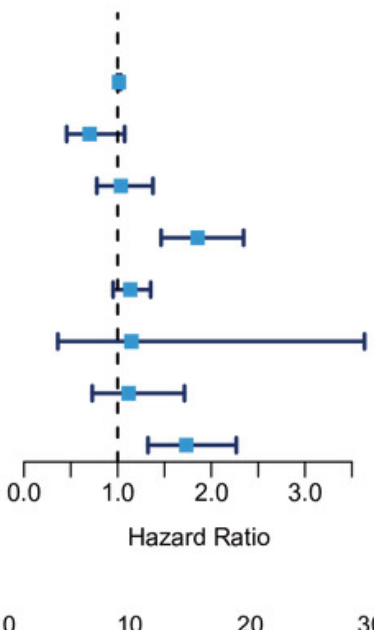

40

50

60

$\begin{array}{r}70 \\ 1 \\ \hline\end{array}$

80

90

100

$$
\begin{array}{llll}
15 & 35 & 55 & 75
\end{array}
$$

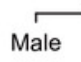

G2 G4

$\mathrm{G} 1 \mathrm{G} 3$

\begin{tabular}{lcc} 
& Stage II & Stage IV \\
Stage I & \multicolumn{1}{l}{ Stage III }
\end{tabular}

1-year survival

3-year survival

\begin{tabular}{lllllllll}
\hline 0 & 0.5 & 1 & 1.5 & 2 & 2.5 & 3 & 3.5 & 4
\end{tabular}

5-year survival

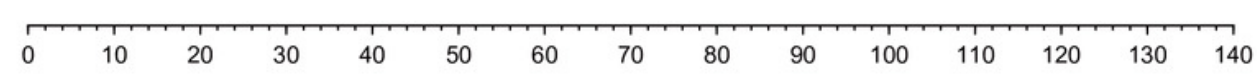

D

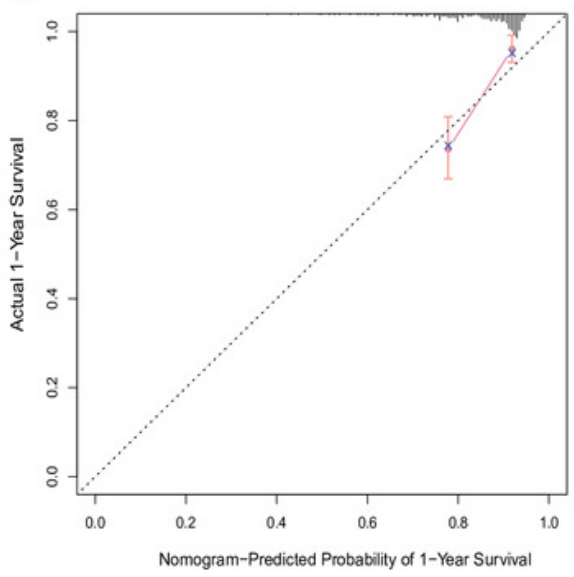

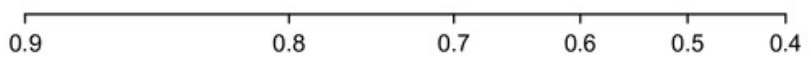
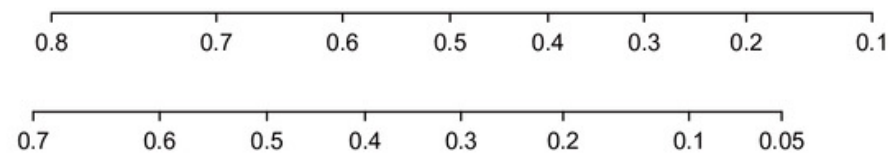

E

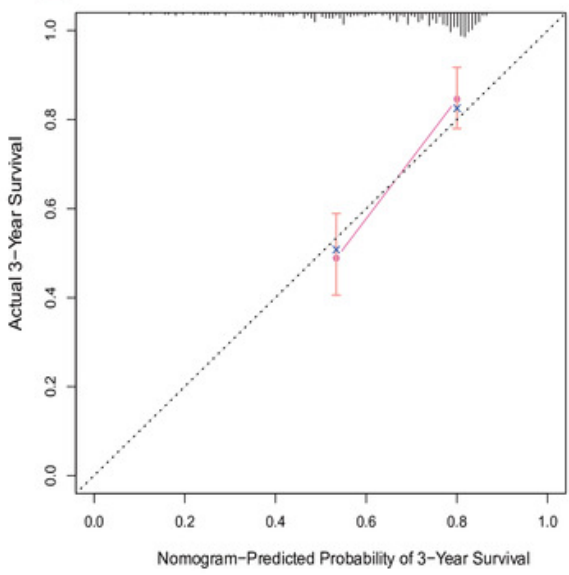

$\mathrm{F}$

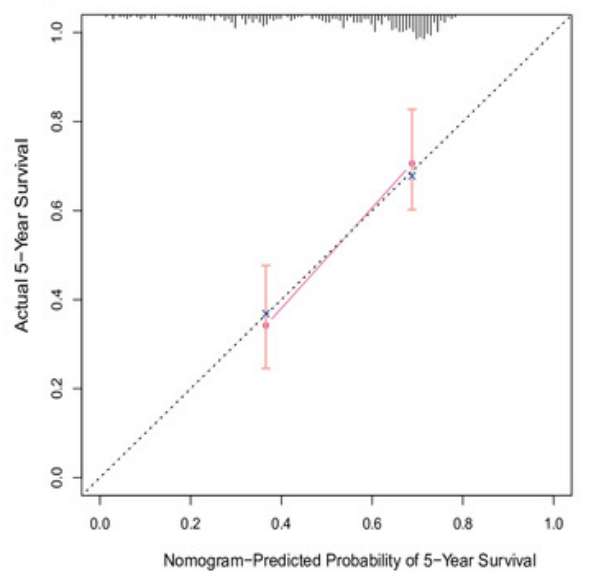




\section{Figure 5}

The survival curves of the FRIncRNAs signature stratified by age, grade, stage, gender, etiology, radiotherapy, and family history.

$(A) \geq 65$ years, (B) < 65 years, (C) grade 1-2, (D) grade 3-4, (E) stage 1-2, (F) stage 3-4, (G) Alcoholic liver disease, (H) NAFLD, (I) no family history, (J) male, (K) HBV positive, (L) HBV and HCV negative, (M) no radiotherapy patients. 
A

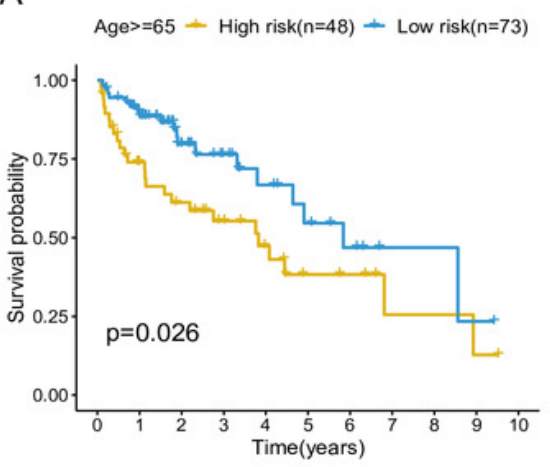

D

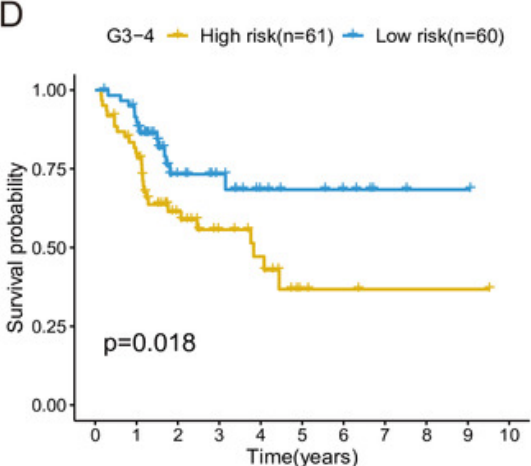

G

Alcoholic liver disease - High risk $(n=41)-$ Low risk $(n=70)$

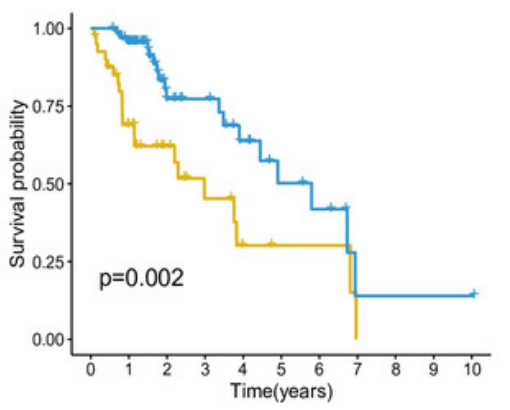

B

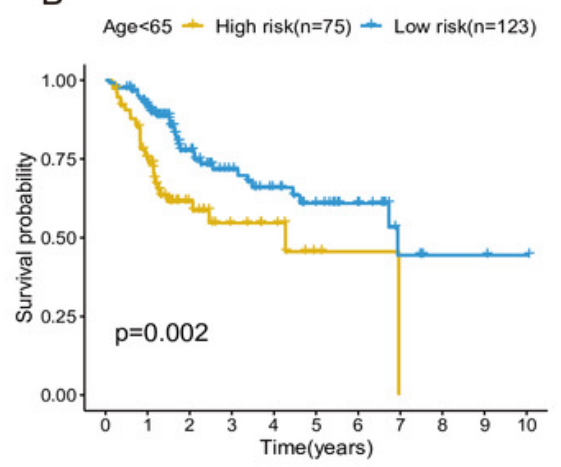

E

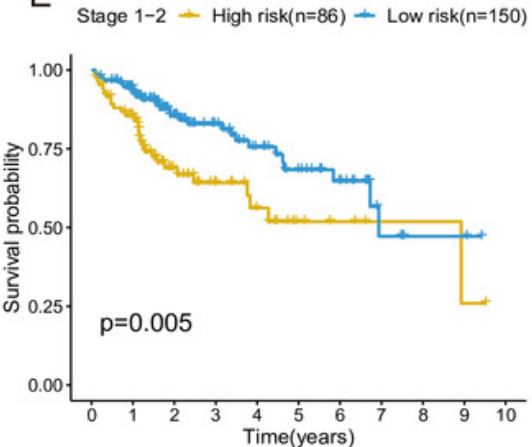

$\mathrm{H}$

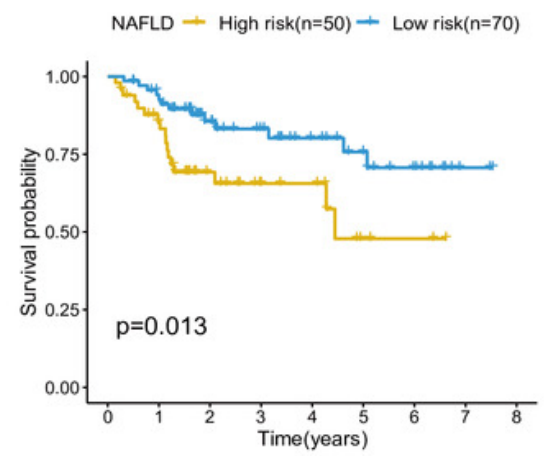

C $\mathrm{G1}-2$ - High risk $(n=62)-$ Low risk $(n=136)$

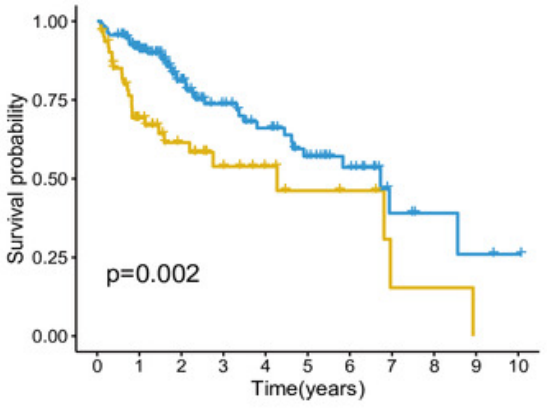

F

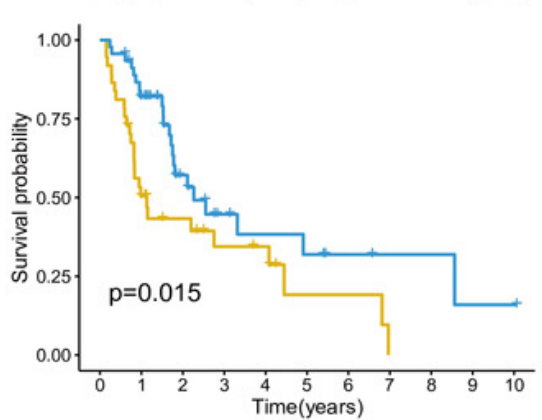

I

Family history $(-)+$ High risk(n=79) - Low risk(n=115)

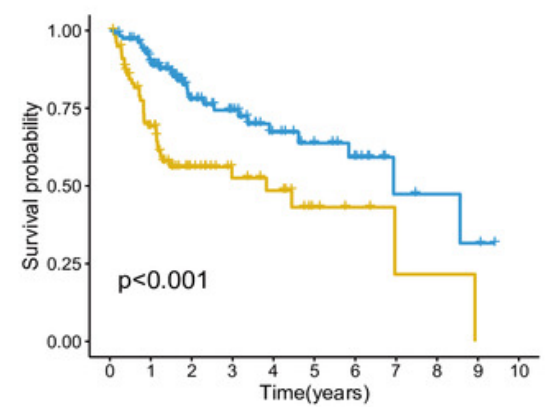

$J$

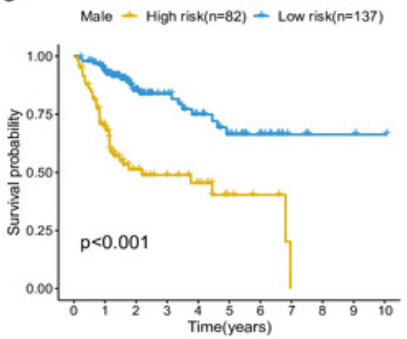

$\mathrm{K}$

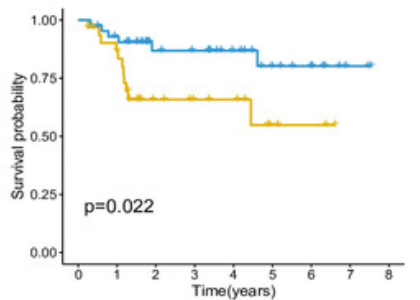

L

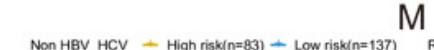

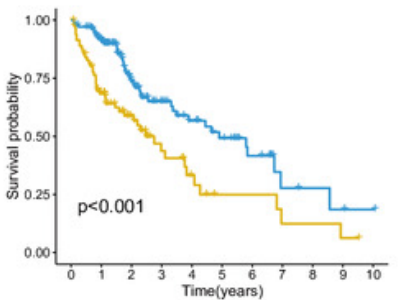
Radiotherapy(-) - High risk(n=128) - Low risk(n=189)

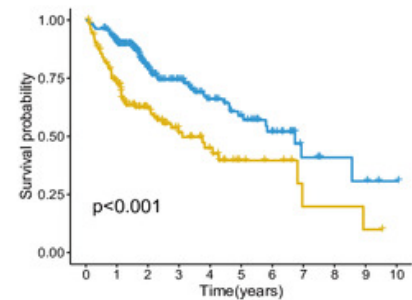




\section{Figure 6}

The verification of prognostic FRIncRNAs signature in validation cohort.

(A) The KM curves of validation cohort revealed that high-risk group had statistical

differences on OS period compared with low-risk group $(P<0.05)$. Univariate $(B)$ and

multivariate (C) COX regression for the FRIncRNAs signature established by training cohort.

(D) AUC of ROC curves validated the predicted performance of signature in validation cohort. 
Risk + High risk + Low risk

A
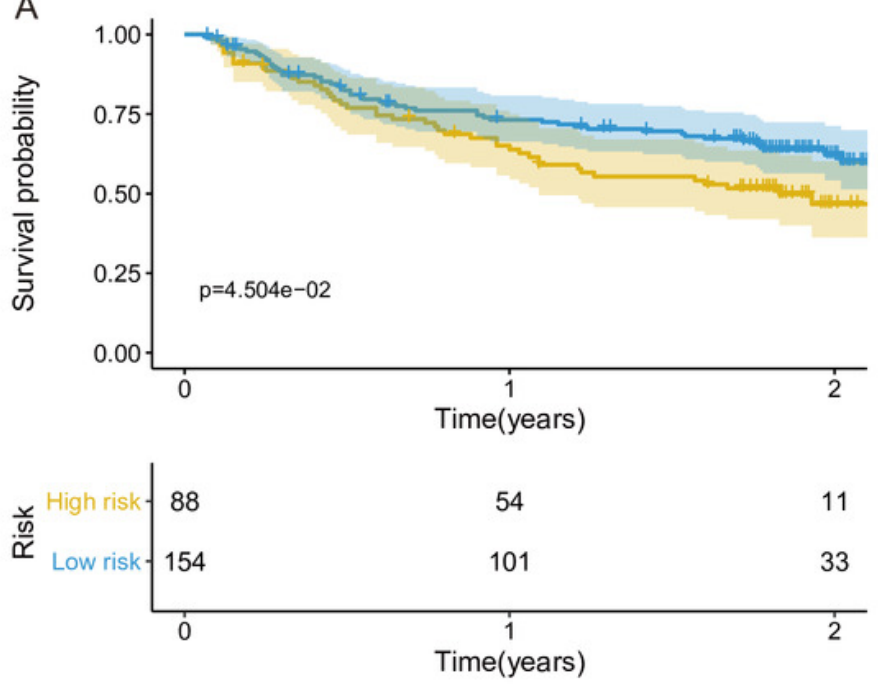

C

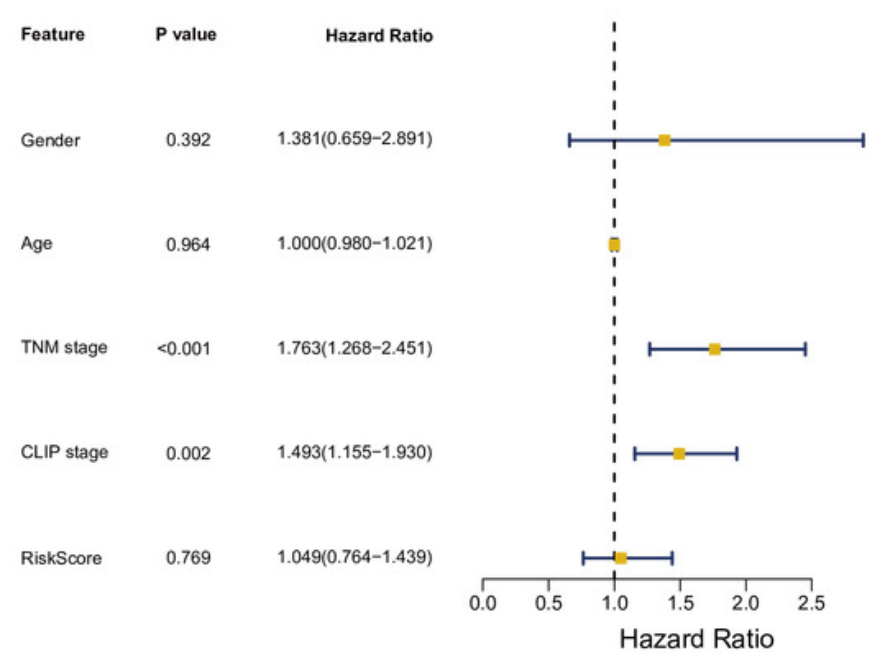

B

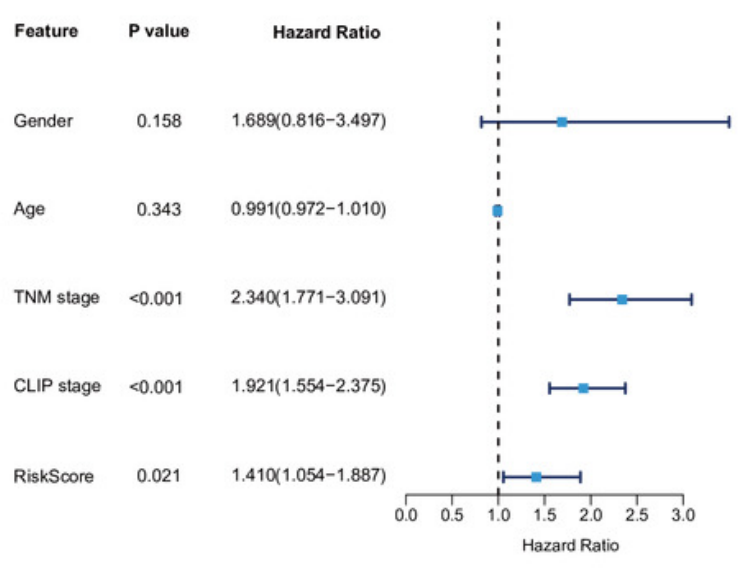

D

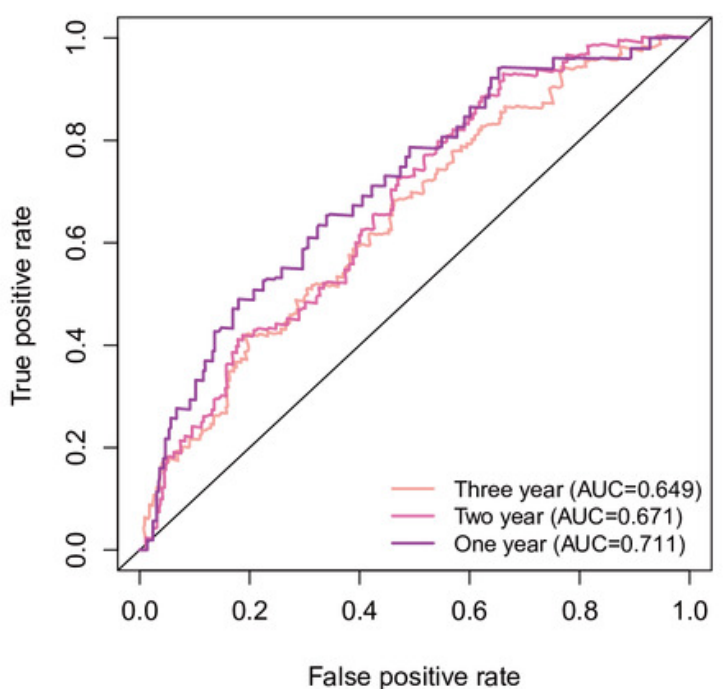


Figure 7

Some cancer-related hallmarks and immunologic features regulated through the FRIncRNAs signature.

(A) Cancer hallmarks. (B) Immunologic signatures. 
A
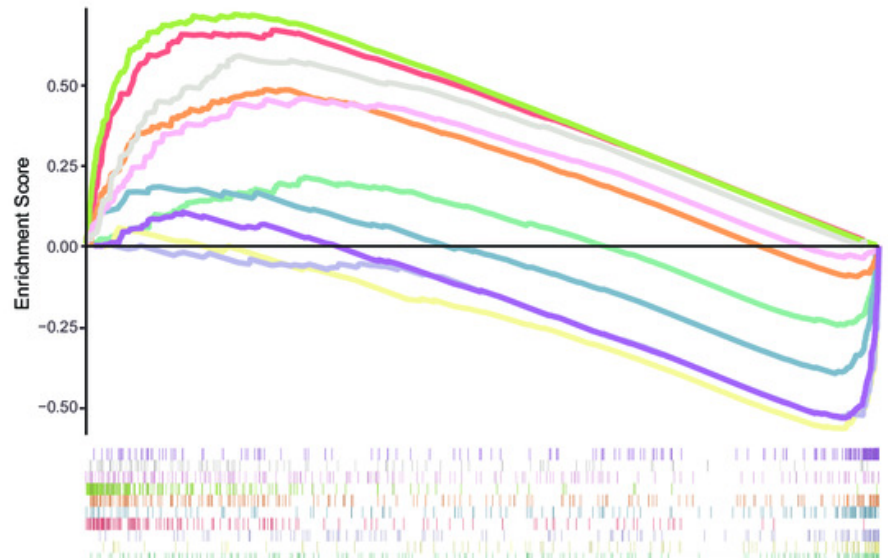

High risk<-----------> Low risk

- HALLMARK_ADIPOGENESIS_IOW - HALLMARK_DNA_REPAIR_high

HALLMARK_BILE_ACID_METABOLISM_low - HALLMARK_GLYCOLYSIS_high

- HALLMARK_COAGULATION_IOW - HALLMARK_MYC_TARGETS_V1_high

- HALLMARK_COAGULATION_JOW
- HALLMARKFATTYACID_METABOLISM_low
- HALLMARK_XENOBIOTIC_METABOLISM_Jow
B

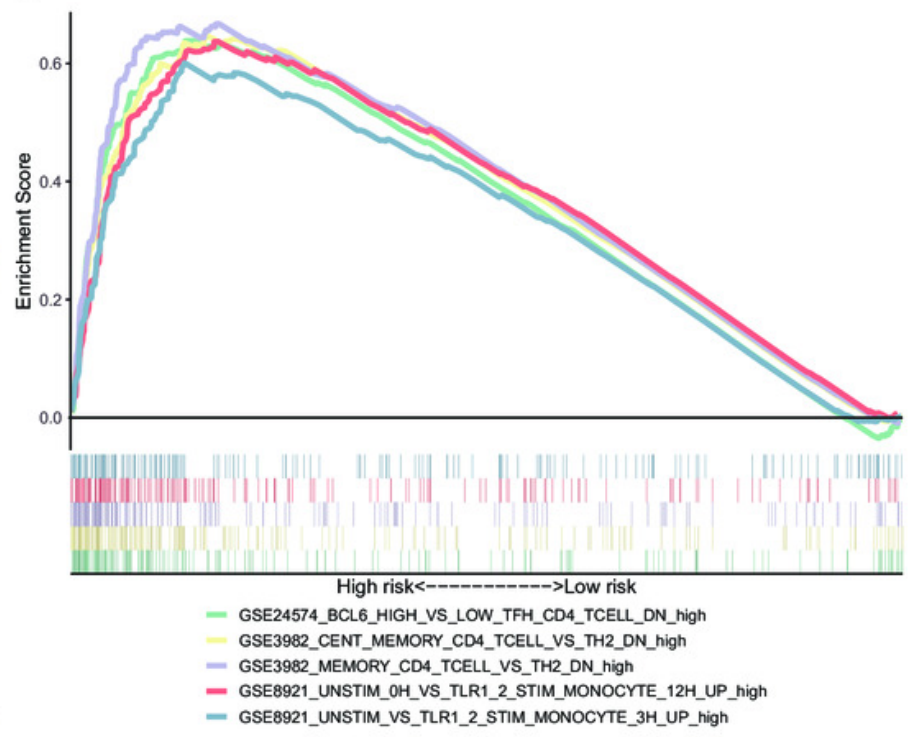




\section{Figure 8}

The relationship between FRIncRNAs signature and TIICS, ICB molecules based on TIMER results.

(A) Spearman correlation between the signature and B cell; (B) Spearman correlation between the signature and Macrophage cell. (C) Spearman correlation between the signature and Myeloid dendritic cell. (D) Spearman correlation between the signature and Neutrophil cell. (E) Spearman correlation between the signature and CD4+ T cell. (F) Spearman correlation between the signature and CD8 $+\mathrm{T}$ cell. (G-I) Significant positive association between our FRIncRNAs signature and ICB receptors PD-1 ( $R=0.17 ; P=0.0019), C T L A-4$ $(R=0.19 ; P<0.001)$, and TIM-3 $(R=0.16 ; P<0.001)$. (J-L) The comparison of the expression levels of PD-1, CTLA-4, and TIM-3 between high-risk and low- groups. 
A

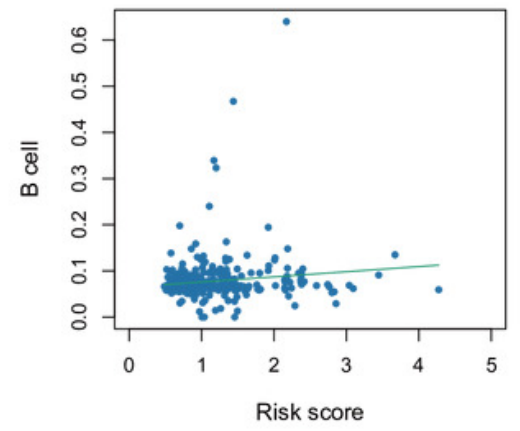

D

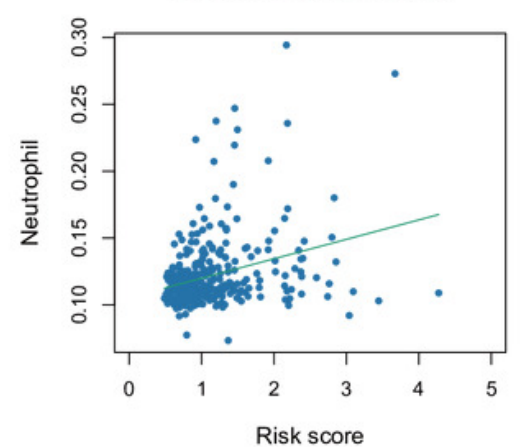

G

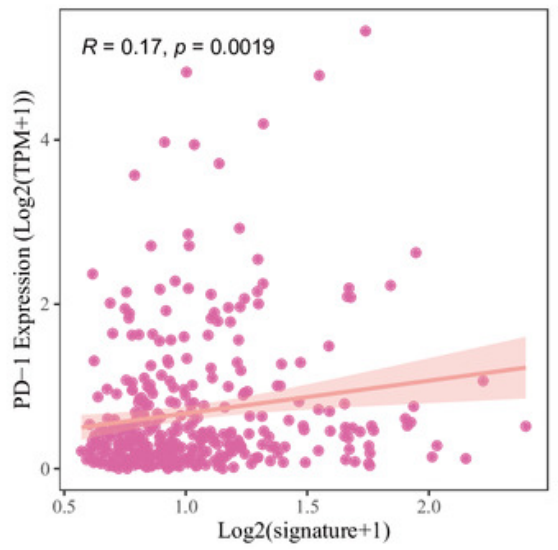

J

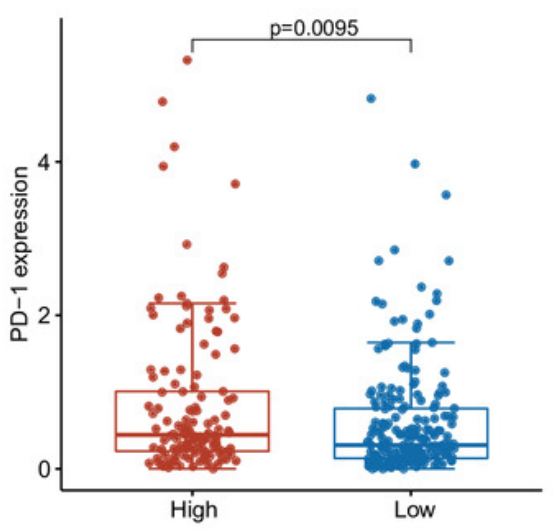

B

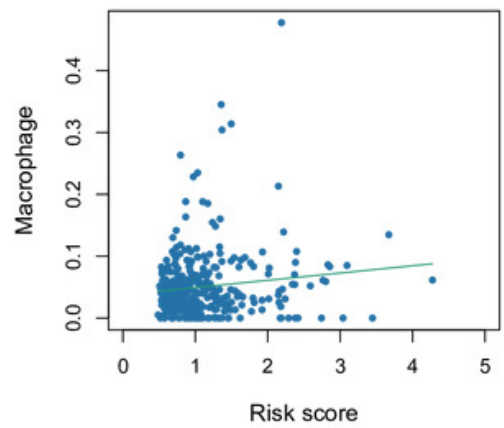

E

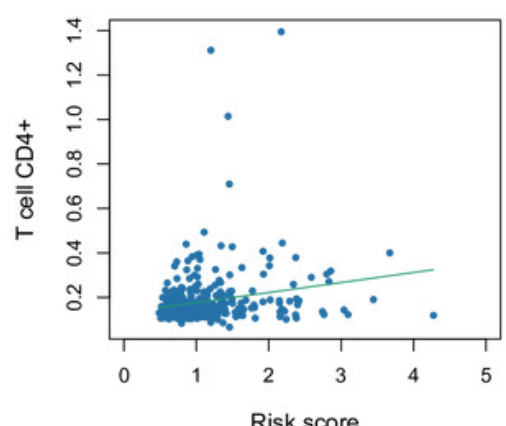

$\mathrm{H}$

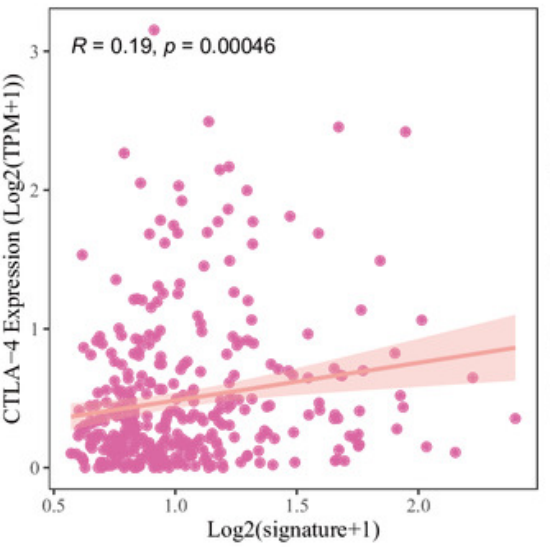

K

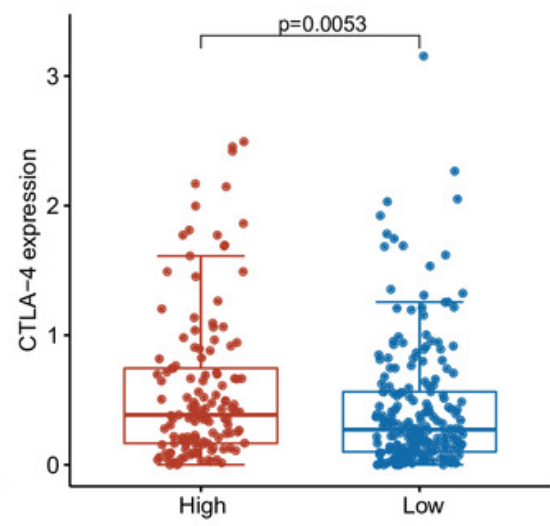

C Cor $=0.268(p=4.662 e-07)$

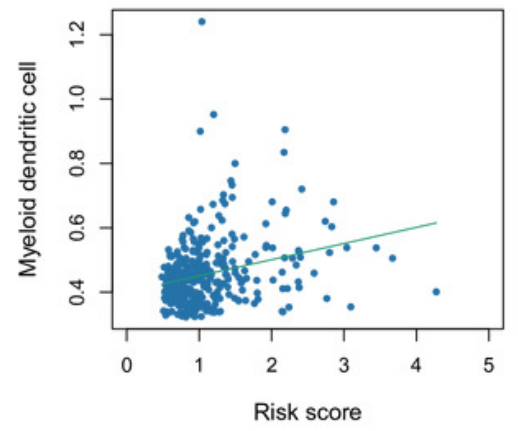

F

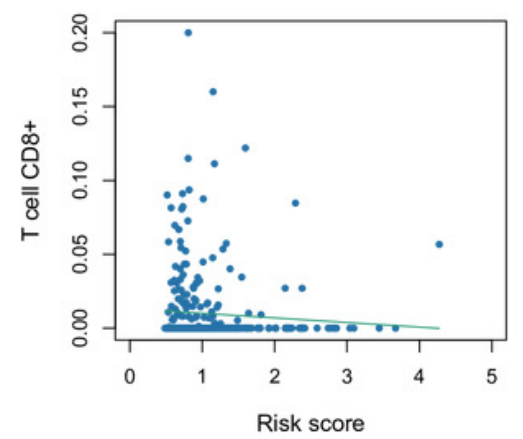

I

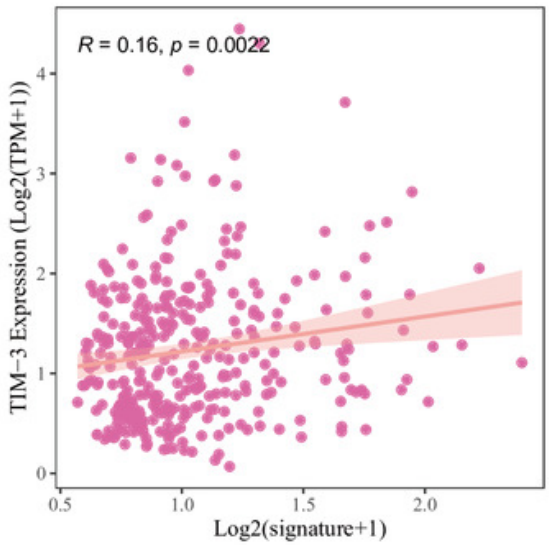

L

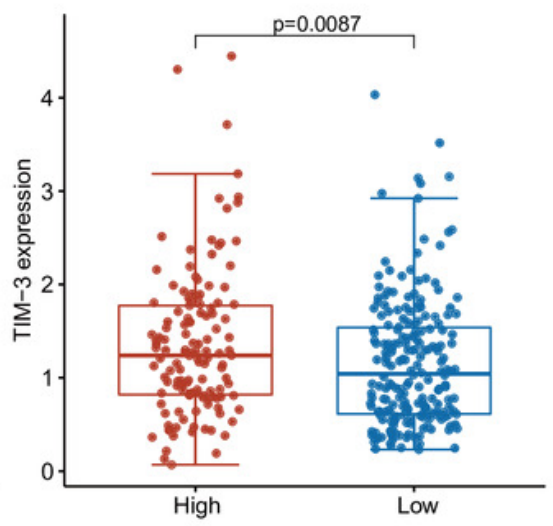


Figure 9

Based on CIBERSORT, EPIC, ESTIMATE, MCP counter, QUANTISEQ, SSGSEA and TIMER algorithms, heatmap of immune infiltration in high- and low-risk group. 


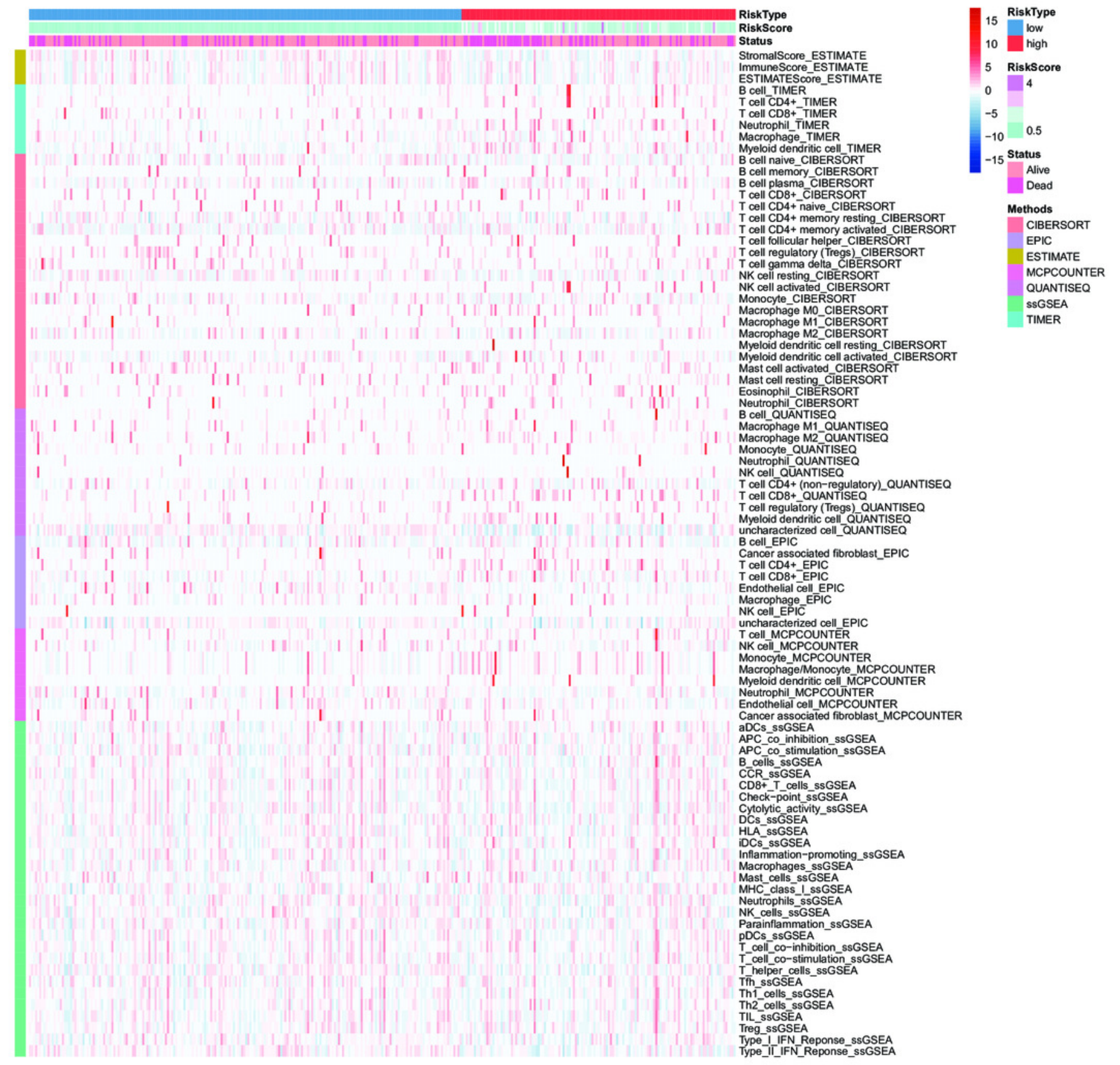




\section{Table 1 (on next page)}

Clinical characteristics of the patients with HCC from TCGA cohort in this study. 


\begin{tabular}{|c|c|c|}
\hline Variables & Number of patients & Percent (\%) \\
\hline \multicolumn{3}{|l|}{ Age } \\
\hline$<65$ & 224 & 59.42 \\
\hline$>=65$ & 152 & 40.32 \\
\hline NA & 1 & 0.27 \\
\hline \multicolumn{3}{|l|}{ Gender } \\
\hline Male & 255 & 67.64 \\
\hline Female & 122 & 32.36 \\
\hline \multicolumn{3}{|l|}{ Grade } \\
\hline G1 & 55 & 14.59 \\
\hline $\mathrm{G} 2$ & 180 & 47.75 \\
\hline G3 & 124 & 32.89 \\
\hline G4 & 13 & 3.45 \\
\hline NA & 5 & 1.33 \\
\hline \multicolumn{3}{|l|}{ Stage } \\
\hline stage I & 175 & 46.42 \\
\hline stage II & 88 & 23.34 \\
\hline stage III & 86 & 22.81 \\
\hline stage IV & 6 & 1.59 \\
\hline NA & 22 & 5.84 \\
\hline \multicolumn{3}{|l|}{ Etiology } \\
\hline Alcohol Liver Disease & 118 & 31.30 \\
\hline NAFLD & 12 & 3.18 \\
\hline $\mathrm{HBV} / \mathrm{HCV}$ & 118 & 31.30 \\
\hline Hemochromatosis & 5 & 1.33 \\
\hline $\mathrm{NA}$ & 124 & 32.89 \\
\hline \multicolumn{3}{|l|}{ Radiotherapy } \\
\hline YES & 8 & 2.12 \\
\hline NO & 338 & 89.66 \\
\hline NA & 31 & 8.22 \\
\hline \multicolumn{3}{|l|}{ Family history } \\
\hline YES & 114 & 30.24 \\
\hline NO & 212 & 56.23 \\
\hline NA & 51 & 13.53 \\
\hline
\end{tabular}

1 Note. NAFLD: nonalcoholic fatty liver disease; HBV: hepatitis B virus; HCV: hepatitis C virus. 


\section{Table 2 (on next page)}

Univariate and multivariate independent prognosticanalysis of FRIncRNAs signature in predicting patient survival. 


\begin{tabular}{ccccc}
\hline & \multicolumn{2}{c}{ Univariate Cox model } & \multicolumn{2}{c}{ Multivariate Cox model } \\
\cline { 2 - 5 } Variables & HR $(95 \% \mathrm{Cl})$ & $p$ value & HR $(95 \% \mathrm{Cl})$ & $p$ value \\
\hline Training cohort & & & & \\
Age & $1.014(0.997-1.032)$ & 0.104 & $1.012(0.994-1.031)$ & 0.181 \\
Gender & $0.701(0.457-1.075)$ & 0.103 & $0.912(0.555-1.500)$ & 0.718 \\
Grade & $1.035(0.778-1.376)$ & 0.815 & $0.971(0.707-1.333)$ & 0.856 \\
Stage & $1.853(1.464-2.345)$ & $<0.001$ & $1.807(1.412-2.313)$ & $<0.001$ \\
Etiology & $1.135(0.952-1.352)$ & 0.158 & $1.041(0.856-1.265)$ & 0.688 \\
Radiotherapy & $1.147(0.362-3.636)$ & 0.815 & $1.060(0.327-3.440)$ & 0.922 \\
Family history & $1.117(0.728-1.713)$ & 0.613 & $0.960(0.602-1.531)$ & 0.863 \\
Risk Score & $1.732(1.324-2.267)$ & $<0.001$ & $1.721(1.280-2.314)$ & $<0.001$ \\
Validation cohort & & & & \\
Gender & $1.689(0.816-3.497)$ & 0.158 & $1.381(0.659-2.891)$ & 0.392 \\
Age & $0.991(0.972-1.010)$ & 0.343 & $1.000(0.980-1.021)$ & 0.964 \\
TNM stage & $2.340(1.771-3.091)$ & $<0.001$ & $1.763(1.268-2.451)$ & $<0.001$ \\
CLIP stage & $1.921(1.554-2.375)$ & $<0.001$ & $1.493(1.155-1.930)$ & 0.002 \\
Risk Score & $1.410(1.054-1.887)$ & 0.021 & $1.049(0.764-1.439)$ & 0.769 \\
\hline
\end{tabular}

